\title{
The Efficacy and Safety of Chinese Herbal Medicine in the Treatment of Knee Osteoarthritis: An Updated Systematic Review and Meta-Analysis of 56 Randomized Controlled Trials
}

\author{
Zhou Lin $\mathbb{D}^{1},{ }^{1}$ Junju Zheng $\mathbb{D},{ }^{1}$ Mangmang Chen $\mathbb{D}^{\mathbb{1}},{ }^{1}$ Jiaru Chen $\mathbb{D}^{1},{ }^{1}$ and Jiejun Lin $\mathbb{D}^{2}$ \\ ${ }^{1}$ Department of Orthopaedic Surgery, Wenzhou Center Hospital, Wenzhou, China \\ ${ }^{2}$ Department of Gastroenterology, Wenzhou Center Hospital, Wenzhou, China \\ Correspondence should be addressed to Jiejun Lin; ljjwzchospital@126.com
}

Received 19 September 2021; Accepted 24 November 2021; Published 7 January 2022

Academic Editor: Elena Azzini

Copyright (C) 2022 Zhou Lin et al. This is an open access article distributed under the Creative Commons Attribution License, which permits unrestricted use, distribution, and reproduction in any medium, provided the original work is properly cited.

\begin{abstract}
Objective. This systematic review and meta-analysis were performed to investigate the efficacy and safety of Chinese herbal medicine (CHM) in the treatment of knee osteoarthritis (KOA). Methods. An electronic search was conducted in eight databases (PubMed, EMBASE, Web of Science, Cochrane Library, Chinese National Knowledge Infrastructure, Chinese Biomedical Literature Database, Chinese VIP Database, and Wanfang Database) from inception until December 2019. The risk of bias assessment of the included RCTs was evaluated by Cochrane collaboration's tool. The inclusion criteria were RCTs that investigated the efficacy and safety of CHM in the treatment of KOA, with no restrictions on publication status or language. The exclusion criteria included nonrandomized or quasi-RCTs, no clear KOA diagnostic approach, combined Chinese medicinal herbs with other traditional Chinese medicine treatment modalities, and published using repeated data and missing data. We computed the relative risk (RR) and the standard mean difference (SMD) for dichotomous outcomes and continuous outcomes, respectively. When heterogeneity was detected or there was significant statistical heterogeneity $\left(P<0.05\right.$ or $I^{2}>50 \%$ ), a random-effects model was employed, followed by further subgroup analysis and metaregression estimations to ascertain the origins of heterogeneity. Otherwise, we used a fixed-effects model $\left(P \geq 0.05\right.$ or $\left.I^{2} \leq 50 \%\right)$. The primary outcome measures were visual analog score (VAS), Western Ontario and McMaster Universities Osteoarthritis Index (WOMAC), Lysholm score, and Lequesne index. Secondary outcome measures were the total clinical effective rate and adverse events. The meta-analysis was performed using the Stata 14.0 software. Results. A total of 56 RCTs comprising 5350 patients met the inclusion criteria. This meta-analysis showed that application of CHM as adjuvant therapy or monotherapy for KOA can significantly decrease VAS, WOMAC, and the Lequesne index and improve the Lysholm score as well as the total effective rate. In addition, this treatment has fewer adverse effects, suggesting that CHM is generally safe and well tolerated among patients with KOA. Conclusion. Our study offers supportive evidence that CHM, either adjuvant therapy or monotherapy, reduces the VAS, WOMAC, and Lequesne index and improves the Lysholm score and overall effective rate in patients with KOA. Additionally, $\mathrm{CHM}$ was well tolerated and safe in KOA patients. We found frequently used CHMs that might contribute to the formulation of a herbal formula that could be considered for further clinical use. However, given the heterogeneity and limited sample size in this study, larger multicenter and high-quality RCTs are needed to validate the benefits of CHM in the treatment of KOA.
\end{abstract}

\section{Introduction}

Knee osteoarthritis (KOA) is a multifactorial degenerative joint disorder characterized by changes in the structure of the joint tissues, including cartilage degeneration, subchondral bone restructuring, and synovial membrane inflamma- tion in the elderly [1]. KOA is more prevalent in older adults [2]. A previous study reported that approximately $12 \%$ of the aging population in the West suffered from $\mathrm{KOA}$, and $25 \%$ of the population above 55 years old had a persistent knee pain episode [3]. According to current data, 9.3 million adults in the US are affected by KOA [4]. As 


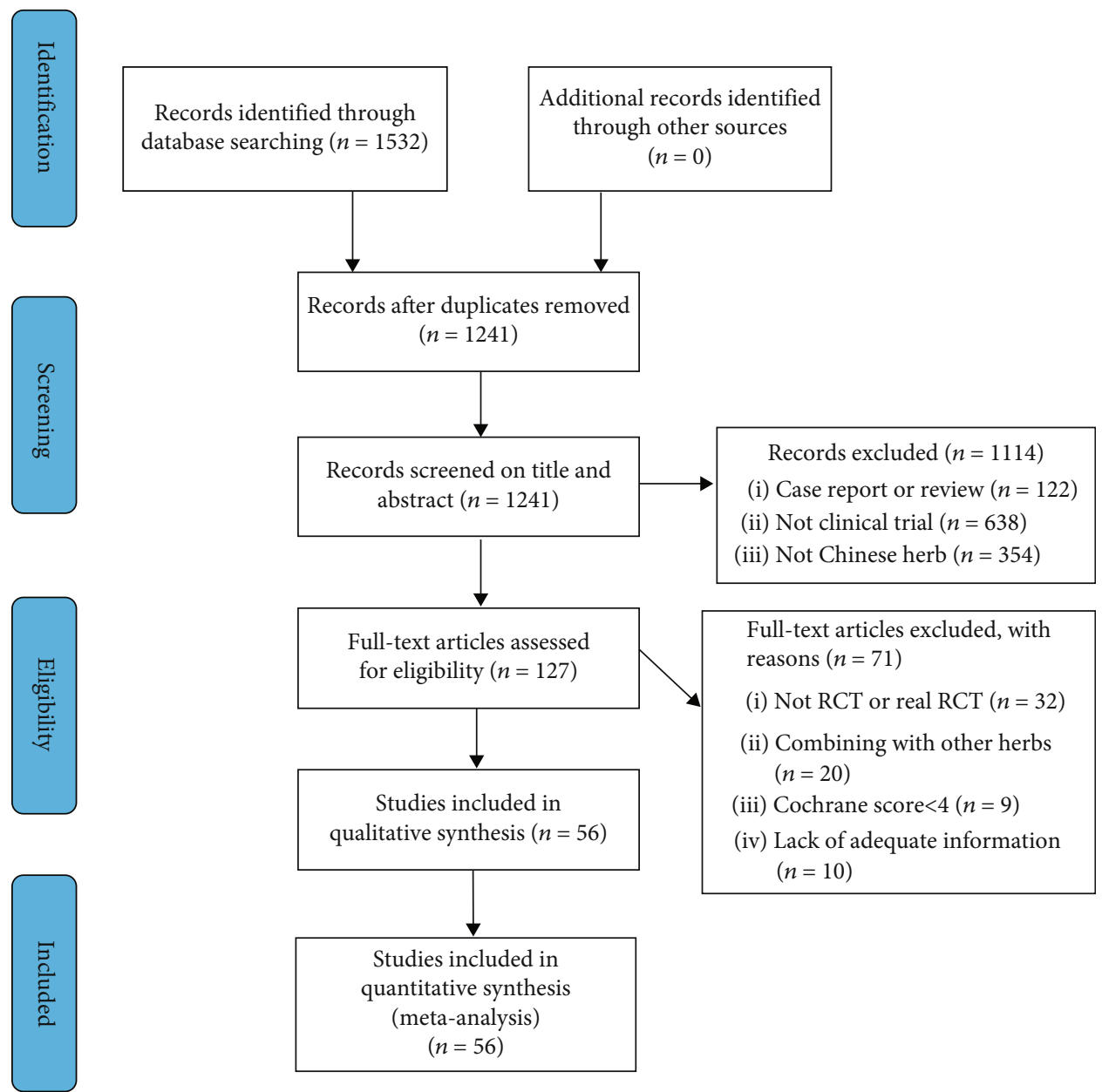

Figure 1: Flowchart of study selection.

the population ages, it is projected that the number of persons with KOA will increase [5, 6]. Osteoarthritis was projected to become the fourth leading cause of disability by 2021 [7].

The primary management goals for KOA have been to alleviate pain, educate patients about the disease, rehabilitate, slow the progression of the disease, and maintain a healthy lifestyle [8]. However, effective therapeutic strategies for KOA disease modification are currently unavailable [9]. The current therapeutic options advanced in various evidence-based clinical guidelines include nonpharmacological therapies, weight loss, oral pharmacological medications, exercise, topical therapies, surgical treatments, and intra-articular therapies [10-12]. Notably, nonsteroidal anti-inflammatory drugs (NSAIDs) and intra-articular hyaluronic acid or corticosteroids are the most frequently used in clinical practice [13-15]. Long-term use of NSAIDs and corticosteroids, on the other hand, has serious adverse effects [16]. Therefore, clinicians and patients are increasingly preferring to treat KOA using complementary as well as alternative medicine [17-19].

Chinese herbal medicine (CHM) has been used in various forms in the treatment of KOA, both in China and the rest of the world $[20,21]$. The adoption of CHM for treating pain disorders, including $\mathrm{KOA}$, has been steadily increasing in Asian countries as well as across the globe [22]. In comparison to other herbal medicines, CHM contains distinct medicinal components that target specific biological processes associated with disease, which are dependent on the differentiation of specific symptoms [23, 24]. According to a recent study, CHM actively reduces pain via analgesic, invigorating blood circulation, and anti-inflammatory effects [25].

$\mathrm{CHM}$ has long been regarded as a vital component in the treatment of KOA in China and is gaining popularity in other parts of the world. However, quantitative research evidence on its effects is currently limited. CHM's biological effect and potential interactions with other prescription medications have not yet been elucidated [26]. Two systematic reviews found that $\mathrm{CMH}$ is both safe and effective in the treatment of $\operatorname{KOA}[27,28]$. However, due to the low quality of the methodology and the limited sample size in the included studies, there is a knowledge gap on the planned application of CHM in treating KOA. Recently, there has been an increase in the number of high-quality randomized controlled clinical trials (RCTs) on the safety and efficacy of $\mathrm{CHM}$ in the treatment of KOA. Therefore, we conducted a large sample size systematic review and meta-analysis of 


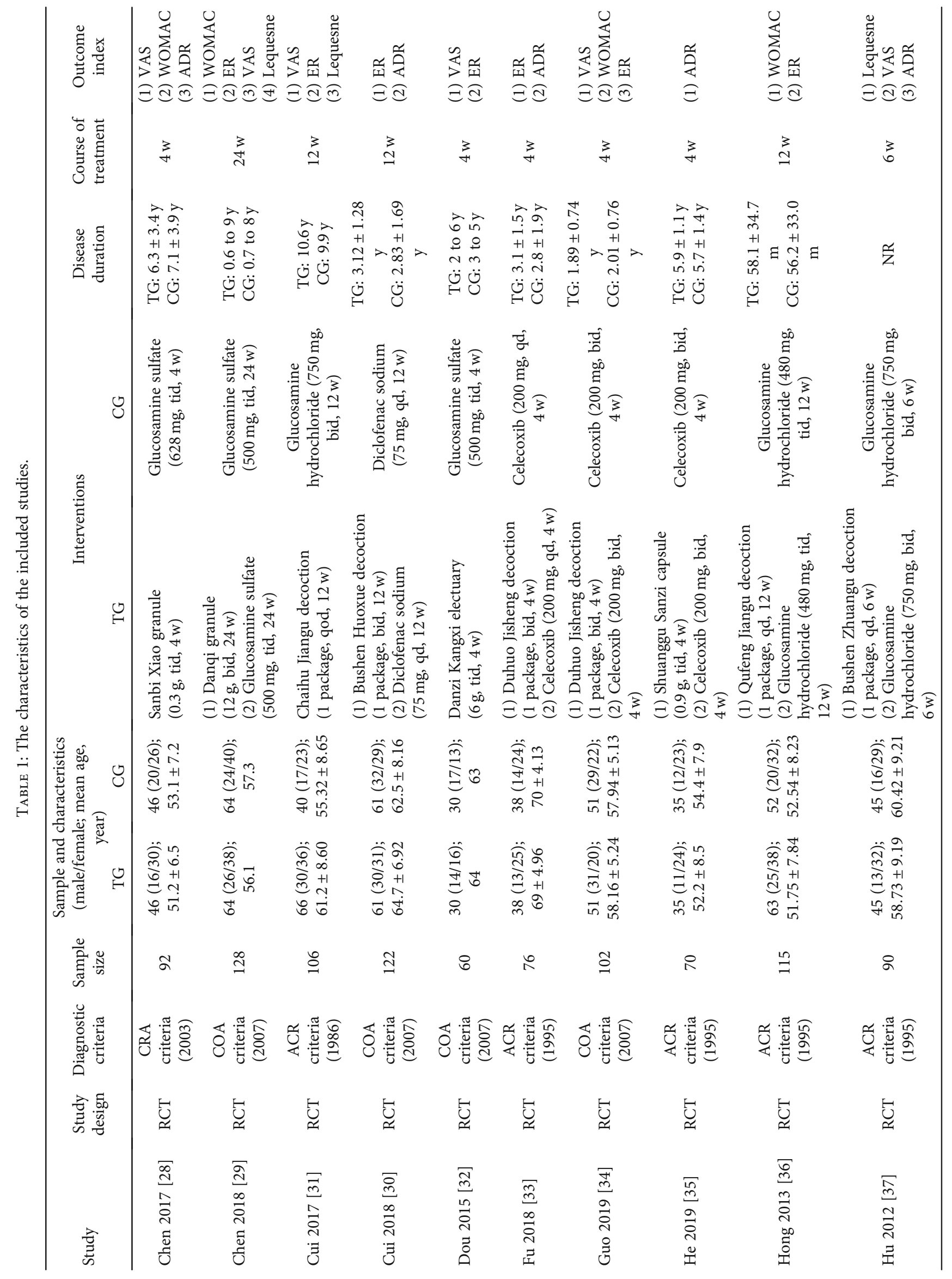




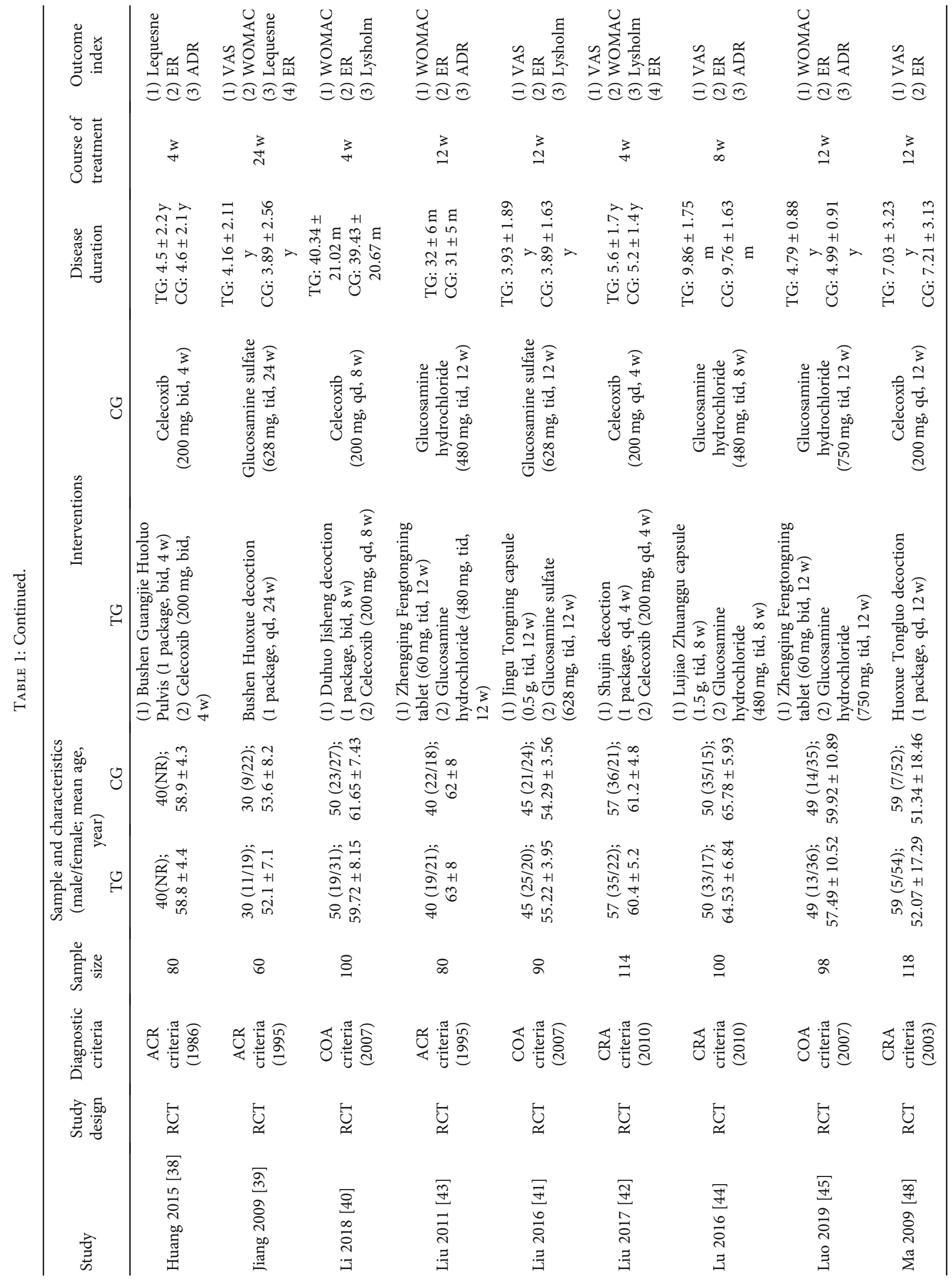




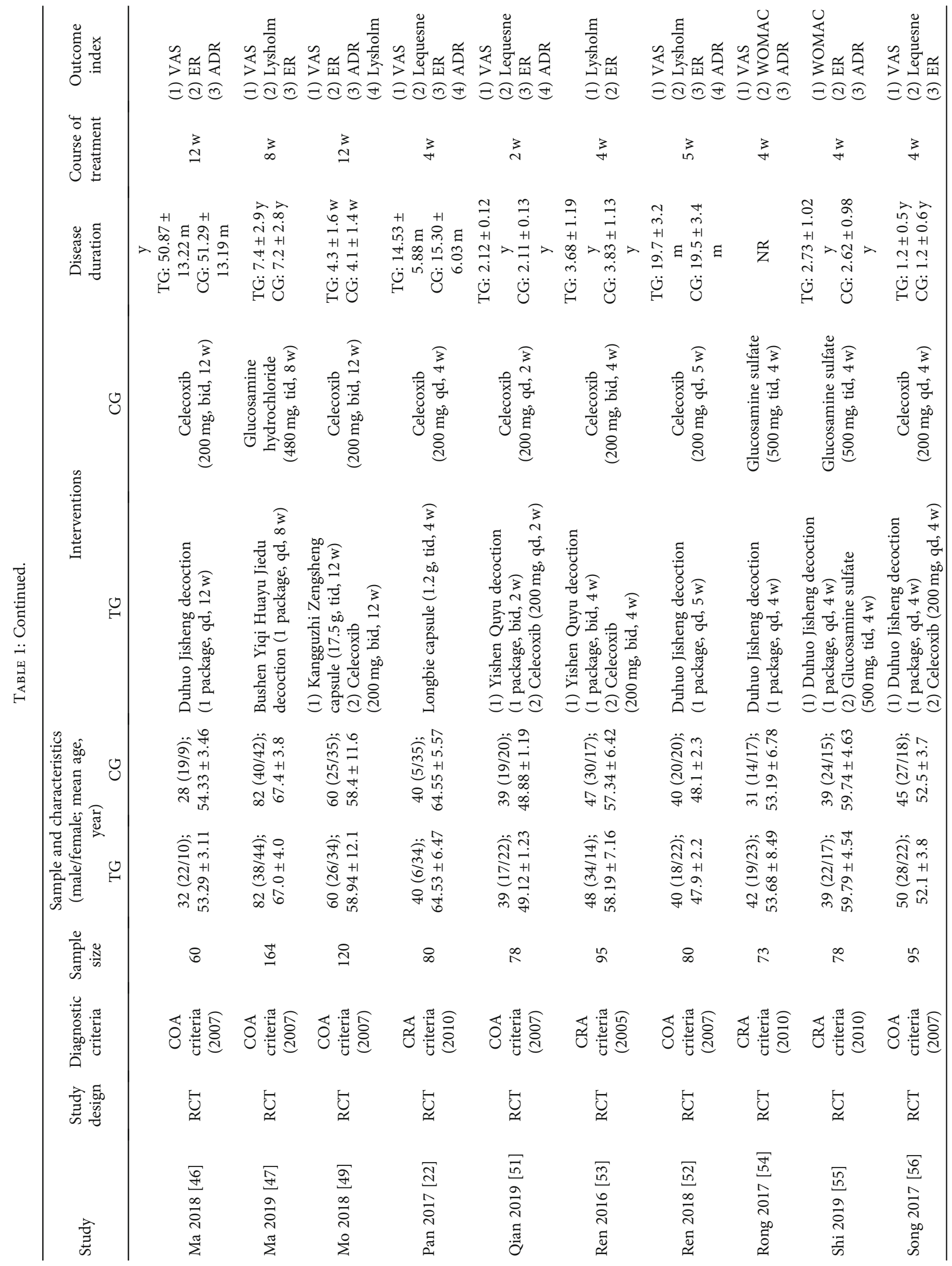




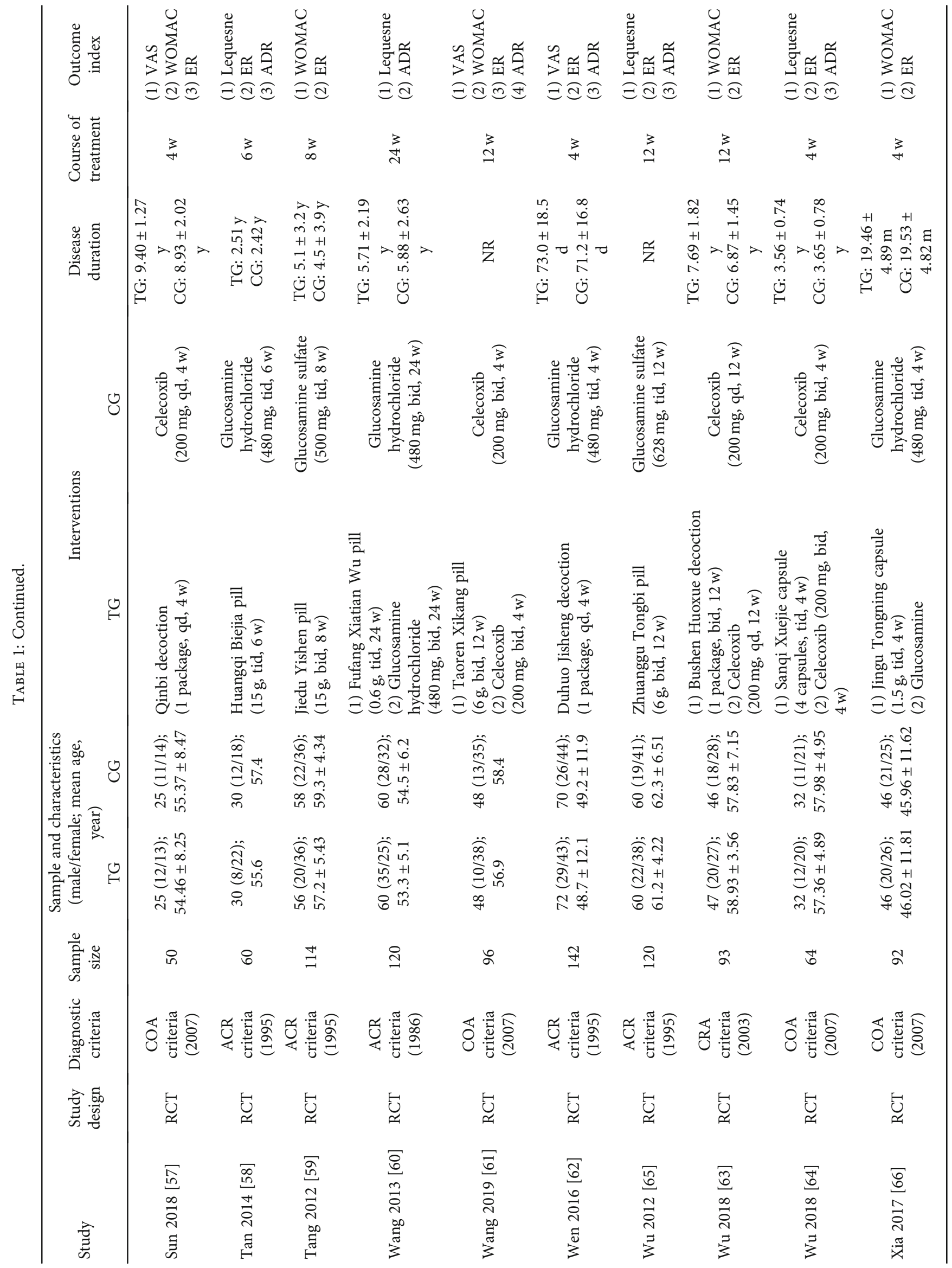




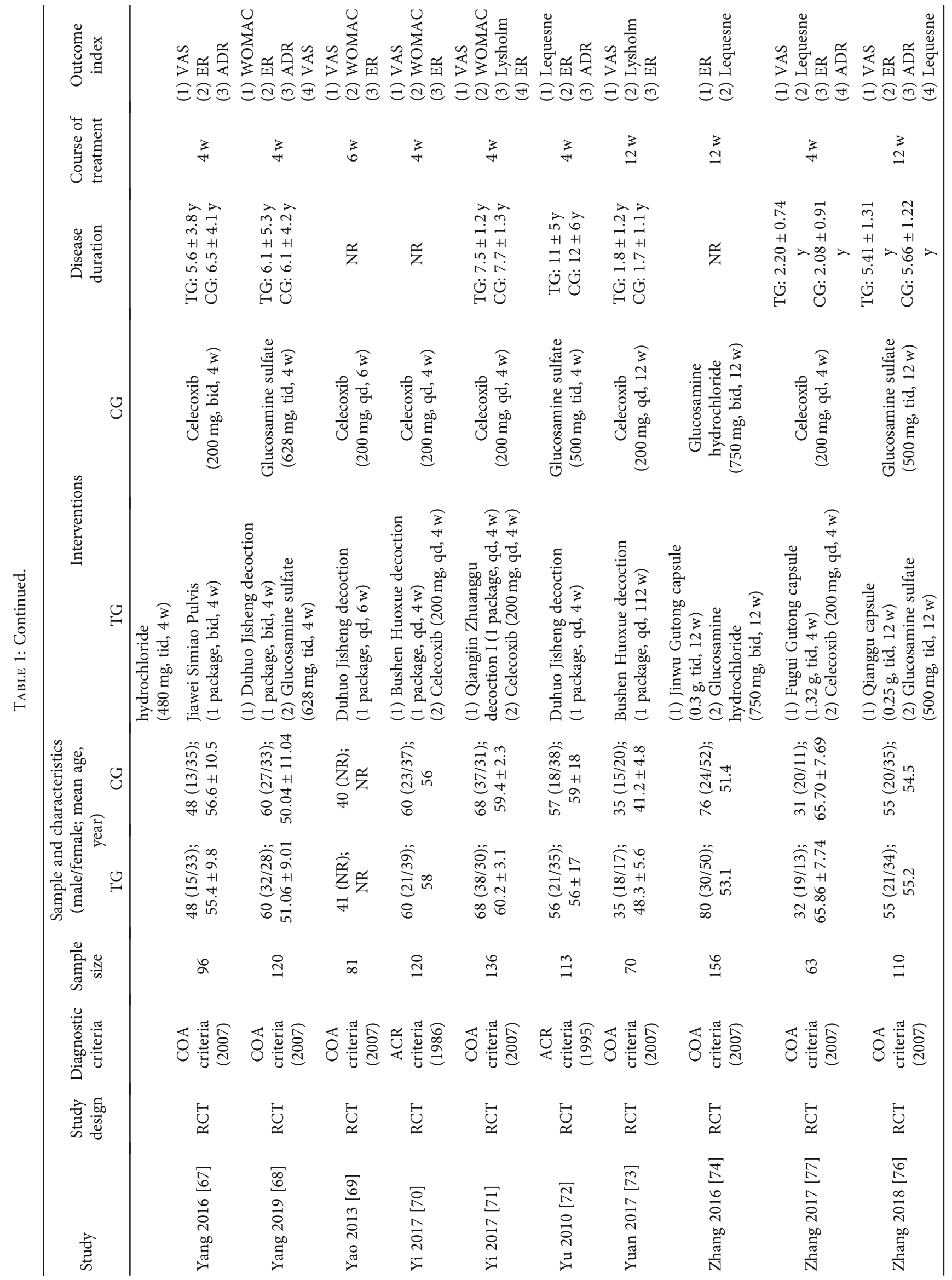




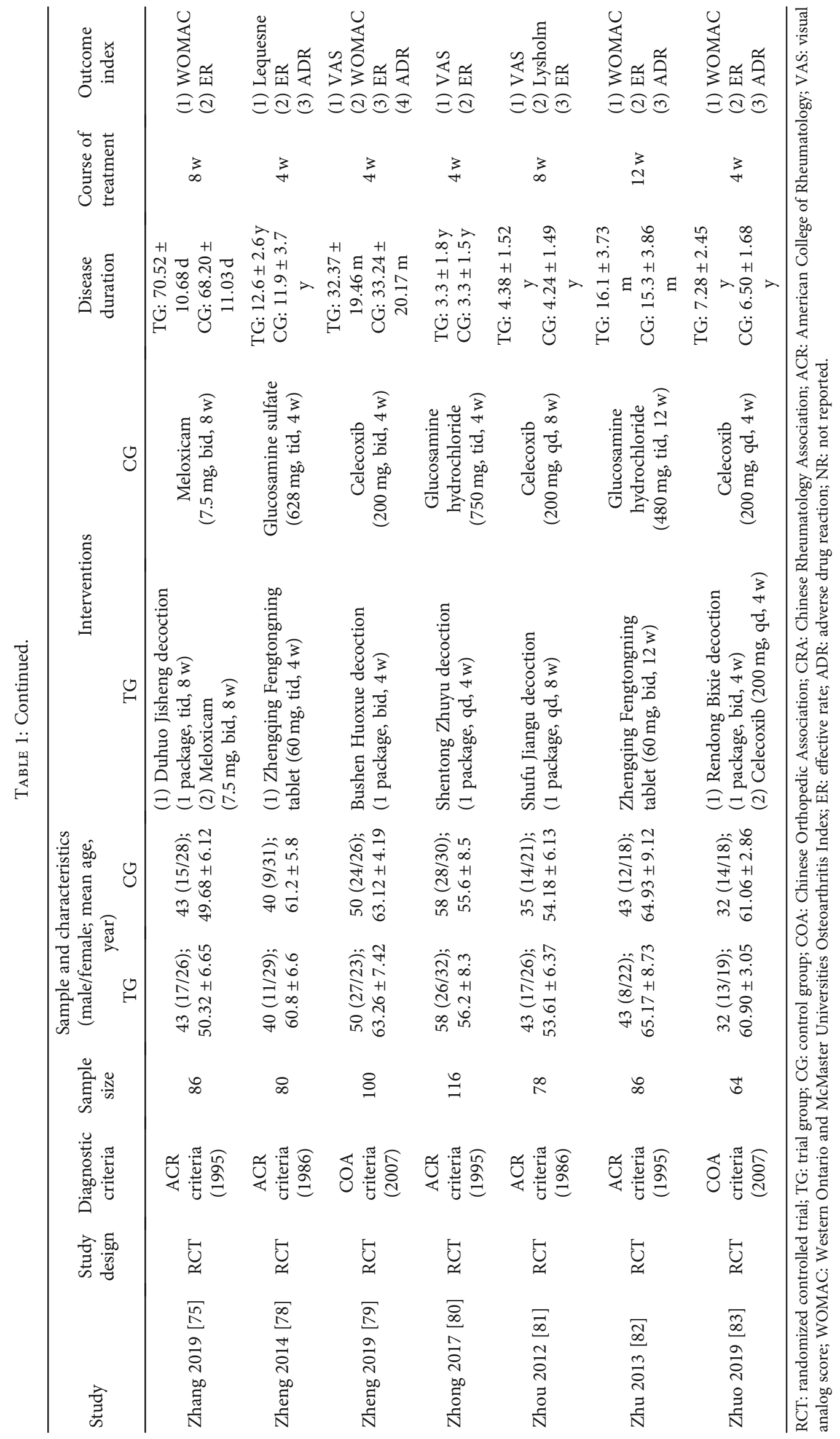


high-quality RCTs focusing on CHM for treating KOA, excluding low-quality studies, in accordance with Cochrane's group guidelines for clinical reviews [29].

\section{Methods}

We used the PRISMA (Preferred Reporting Items for Systematic Reviews and Meta-Analyses) Statement to perform a systematic review and meta-analysis [30]. This study has been registered at http://www.researchregistry.com, and the study's unique identifying number (UIN) from the Research Registry is reviewregistry971. There are no protocols preregistered for this review. We did not collect any primary personal data; hence, we did not require ethical approval.

2.1. Database and Search Strategies. We performed electronic searches in eight repositories from their inception to December 2019: PubMed, Web of Science, EMBASE, Cochrane Library, Chinese Biomedical Literature Database, Chinese National Knowledge Infrastructure, Wanfang Database, and Chinese VIP Database. Additionally, we performed manual searches in the references section of previously published systematic reviews for additional relevant literature. Moreover, the literature search was not limited to any language of publishing. The search criteria used for PubMed were provided as a supplementary material (available here) and were appropriately modified for the other databases.

\subsection{Eligibility Criteria}

2.2.1. Types of Studies. We included RCTs that investigated the efficacy and safety of CHM in the treatment of KOA, with no restrictions on publication status or language. If we discovered a relevant study with three treatment arms, we only retrieved data for the CHM arm(s) and the control $\operatorname{arm}(\mathrm{s})$. We excluded quasirandomized trials, such as studies in which subjects were allocated based on their date of birth, as well as the order in which they were admitted.

2.2.2. Participant Types. We included subjects diagnosed with KOA based on the Chinese Orthopedic Association (COA) criteria (2007), American College of Rheumatology (ACR) criteria (1986 or 1995), and Chinese Rheumatology Association (CRA) criteria (2003, 2005, or 2010) regardless of disease course and severity, age, or gender.

2.2.3. Types of Interventions. Regardless of the dosage, duration, administration route, administration techniques, or duration of therapy, the evaluated therapeutic intervention constituted CHM as monotherapy or a complement to western conventional medicine (WCM). The control group received WCM either alone or in combination with placebo. We excluded trials in which multiple types of CHM therapies were compared.

2.2.4. Outcome Measure Types. The primary outcome parameters included the following: (1) visual analog score (VAS), (2) Western Ontario and McMaster Universities Osteoarthritis Index (WOMAC), (3) Lysholm score, and (4) Lequesne index. The secondary outcome parameters included the following: (1) the overall clinical effectiveness rate and (2) adverse events.
2.3. Literature Selection. The PRISMA flow diagram was used to select the trials that were included. We imported the literature results into the Endnote X7 software. Two independent authors initially screened the titles and abstracts of potentially eligible articles to remove duplications as well as RCTs that did not meet the inclusion criteria. Following that, we downloaded and reviewed the full texts of the remaining prospective studies. Any disagreements between the two authors were resolved through discussion with a third independent author.

2.4. Data Extraction. Two independent reviewers extracted the data, while a third independent reviewer checked for consistency. A standard form was used to collect the retrieved items, which included the following basic research information: the name(s) of the author(s), publication date, study design, diagnostic criteria, sample size, age, CHM and WCM intervention methods, gender, disease duration, and course of treatment. We retrieved the mean, standard deviation (SD), and the number of participants in each study for continuous outcomes. For dichotomous outcomes, we retrieved the total number of CHM and WCM events as well as the number of occurrences in each group. Where possible, we recomputed the data in other formats to allow for pooled analysis. Any disagreements that arose between these two reviewers were resolved through dialogue. We contacted the relevant authors of the included studies to provide us with any missing data and additional information.

2.5. Quality Assessment of Included Studies. Two independent authors used the Cochrane collaboration tool to assess the quality of methodology and risk of bias of the included RCT studies [31]. This Cochrane tool assesses the following parameters, randomization, subject blinding, allocation concealment, outcome evaluation blinding, selective outcome reporting, incomplete outcome data, and other bias, and categorizes studies as unclear, low, or high risk of bias for each item.

2.6. CHM Composition. We compiled a list of the major components of the CHM formulae. We determined the frequency of use of all Chinese medicinal herbs and estimated and discussed in detail those that were frequently used.

2.7. Statistical Analysis. The Stata software (version 12.0; StataCorp, College Station, TX) was used to evaluate all of the data retrieved in this study for meta-analysis. When heterogeneity was detected or there was significant statistical heterogeneity $\left(P<0.05\right.$ or $\left.I^{2}>50 \%\right)$, a random-effects model was employed, followed by further subgroup analysis and metaregression estimations to ascertain the origins of heterogeneity. Otherwise, we used a fixed-effects model $\left(P \geq 0.05\right.$ or $\left.I^{2} \leq 50 \%\right)$. We conducted a sensitivity analysis by excluding individual studies one by one to determine the strength and stability of the pooled data. Besides, the effect of publication bias was examined using Begg's and Egger's tests. We computed the relative risk $(R R)$ and the standard mean difference (SMD) for dichotomous outcomes and continuous outcomes, respectively. 
TABle 2: Assessment of study quality and risk of bias.

\begin{tabular}{|c|c|c|c|c|c|c|c|c|}
\hline \multirow{2}{*}{ Study } & \multicolumn{8}{|c|}{ 7-item criteria } \\
\hline & A & B & $\mathrm{C}$ & $\mathrm{D}$ & $\mathrm{E}$ & $\mathrm{F}$ & G & Total \\
\hline Chen 2017 [28] & $?$ & - & $?$ & + & + & + & + & 4 \\
\hline Chen 2018 [29] & + & $?$ & + & + & + & + & + & 6 \\
\hline Cui 2017 [31] & + & $?$ & + & $?$ & + & + & + & 5 \\
\hline Cui 2018 [30] & $?$ & $?$ & + & + & + & + & + & 5 \\
\hline Dou 2015 [32] & + & + & + & + & + & + & + & 7 \\
\hline Fu 2018 [33] & + & - & + & $?$ & + & + & + & 5 \\
\hline Guo 2019 [34] & + & + & - & - & + & + & + & 5 \\
\hline He 2019 [35] & $?$ & $?$ & + & + & + & - & + & 4 \\
\hline Hong 2013 [36] & $?$ & $?$ & + & + & + & + & + & 5 \\
\hline Hu 2012 [37] & $?$ & $?$ & + & + & + & + & + & 5 \\
\hline Huang 2015 [38] & $?$ & - & + & - & + & + & + & 4 \\
\hline Jiang 2009 [39] & $?$ & $?$ & + & + & + & + & $?$ & 4 \\
\hline Li 2018 [40] & $?$ & - & - & + & + & + & + & 4 \\
\hline Liu 2011 [43] & $?$ & $?$ & + & + & + & + & - & 4 \\
\hline Liu 2016 [41] & $?$ & $?$ & + & + & + & + & + & 5 \\
\hline Liu 2017 [42] & $?$ & + & + & + & + & + & + & 6 \\
\hline Lu 2016 [44] & $?$ & $?$ & $?$ & + & + & + & + & 4 \\
\hline Luo 2019 [45] & + & $?$ & + & - & + & - & + & 4 \\
\hline Ma 2009 [48] & $?$ & $?$ & + & + & $?$ & + & + & 4 \\
\hline Ma 2018 [46] & + & + & - & - & + & + & + & 5 \\
\hline Ma 2019 [47] & + & $?$ & + & + & + & + & + & 6 \\
\hline Mo 2018 [49] & + & $?$ & + & $?$ & + & + & + & 5 \\
\hline Pan 2017 [22] & + & - & + & - & + & + & + & 5 \\
\hline Qian 2019 [51] & + & - & + & + & + & + & + & 6 \\
\hline Ren 2016 [53] & $?$ & + & + & + & + & - & + & 5 \\
\hline Ren 2018 [52] & + & $?$ & - & - & + & + & + & 4 \\
\hline Rong 2017 [54] & $?$ & + & - & - & + & + & + & 4 \\
\hline Shi 2019 [55] & + & - & + & + & + & + & + & 6 \\
\hline Song 2017 [56] & + & - & + & + & + & + & + & 6 \\
\hline Sun 2018 [57] & + & - & + & + & + & + & + & 6 \\
\hline Tan 2014 [58] & $?$ & $?$ & + & - & + & + & + & 4 \\
\hline Tang 2012 [59] & + & $?$ & + & $?$ & + & + & + & 5 \\
\hline Wang 2013 [60] & $?$ & $?$ & + & + & + & $?$ & + & 4 \\
\hline Wang 2019 [61] & + & - & $?$ & + & + & + & + & 5 \\
\hline Wen 2016 [62] & + & - & + & + & + & + & + & 6 \\
\hline Wu 2012 [65] & + & + & + & + & + & + & + & 7 \\
\hline Wu 2018 [63] & + & - & - & - & + & + & + & 4 \\
\hline Wu 2018 [64] & + & $?$ & + & $?$ & + & + & + & 5 \\
\hline Xia 2017 [66] & $?$ & $?$ & - & + & + & + & + & 4 \\
\hline Yang 2016 [67] & $?$ & $?$ & - & + & + & + & + & 4 \\
\hline Yang 2019 [68] & + & $?$ & + & - & + & + & + & 5 \\
\hline Yao 2013 [69] & + & $?$ & + & + & + & + & + & 6 \\
\hline Yi 2017 [70] & + & - & + & + & + & + & + & 6 \\
\hline Yi 2017 [71] & $?$ & $?$ & + & - & + & + & + & 4 \\
\hline Yu 2010 [72] & $?$ & - & + & + & + & + & - & 4 \\
\hline Yuan 2017 [73] & + & $?$ & + & $?$ & + & + & + & 5 \\
\hline Zhang 2016 [74] & $?$ & $?$ & + & - & + & + & + & 4 \\
\hline Zhang 2017 [77] & $?$ & - & + & + & + & + & + & 5 \\
\hline
\end{tabular}

TABLE 2: Continued.

\begin{tabular}{lccccccccc}
\hline \multirow{2}{*}{ Study } & \multicolumn{1}{c}{ A } & B & C & D & E & F & G & Total \\
\hline Zhang 2018 [76] & + & - & $?$ & - & + & + & + & 4 \\
Zhang 2019 [75] & + & + & - & - & + & - & + & 4 \\
Zheng 2014 [78] & $?$ & $?$ & + & + & + & - & + & 4 \\
Zheng 2019 [79] & + & + & + & + & + & + & + & 7 \\
Zhong 2017 [80] & + & $?$ & + & + & + & + & + & 6 \\
Zhou 2012 [81] & $?$ & - & + & + & + & + & + & 5 \\
Zhu 2013 [82] & + & - & + & + & + & + & + & 6 \\
Zhuo 2019 [83] & $?$ & - & $?$ & + & + & + & + & 4 \\
\hline
\end{tabular}

A to $G$, the 7-item criteria. A: random sequence generation; B: allocation concealment; C: blinding of participants and personnel; D: blinding of outcome assessment; E: incomplete outcome data; F: selective reporting; G: other bias; +: low risk of bias, -: high risk of bias; ?: uncertain risk of bias.

\section{Results}

3.1. Description of Studies. We identified 1532 potentially relevant hits from the repositories. After eliminating duplicated RCTs, we were left with 1241 peer-reviewed articles. Subsequently, we applied the inclusion and exclusion criteria to the titles and abstracts and eliminated 1114 irrelevant studies. Additionally, after reviewing the full text of the 127 remaining studies, we eliminated 71 studies for failing to meet at least one of the following criteria:

(1) Nonrandomized or quasi-RCTs

(2) No clear KOA diagnostic approach

(3) Combined Chinese medicinal herbs with other TCM treatment modalities

(4) Published using repeated data

(5) Missing data.

Finally, 56 articles [32-87] were included for analysis (Figure 1).

3.2. Characteristics and Quality Assessment of the Eligible Studies. Table 1 summarizes the characteristics of the 56 included RCTs. All the included RCTs were published between 2009 and 2019. All studies enrolled 5350 patients, with 2710 in the treatment arm vs. 2640 in the control arm. Table 2 summarizes the risk of bias analysis conducted on RCTs. Table 3 summarizes the ingredients of CHM used in the included studies.

\subsection{Results of Meta-Analysis}

3.3.1. VAS. On the basis of regarding the VAS, nine studies compared CHM plus NSAIDS with NSAIDS alone [38, 46, $53,55,60,65,75,81]$. The pooled results revealed that CHM plus NSAIDS significantly decreased VAS when compared to NSAIDS alone $(\mathrm{SMD}=-1.990 ; 95 \% \mathrm{CI}=-2.698$ to -1.282 ; $P \leq 0.001$; heterogeneity $\chi^{2}=155.33$, df $=8, I^{2}=94.8 \%, P \leq$ 0.001 ) (Figure 2(a)). Ten studies compared the VAS of CHM and NSAIDS [50, 52, 54, 56, 61, 71, 73, 77, 83, 85]. 
TABLE 3: Ingredients of CHM in the included studies.

\begin{tabular}{|c|c|c|c|c|}
\hline Study & $\begin{array}{l}\text { Prescription } \\
\text { name }\end{array}$ & Ingredients of herb prescription & Preparations & Quality control \\
\hline Chen 2017 [28] & $\begin{array}{l}\text { Sanbi Xiao } \\
\text { granule }\end{array}$ & $\begin{array}{l}\text { Angelica sinensis, Radix Paeoniae Alba, Radix Rehmanniae Recen, } \\
\text { Ginseng, Astragalus, Achyranthes root, Eucommia ulmoides }\end{array}$ & Granule & $\begin{array}{l}\text { Guangdong FDA } \\
\text { approval number: } \\
\text { ZB20060360 }\end{array}$ \\
\hline Chen 2018 [29] & Danqi granule & $\begin{array}{l}\text { Radix Rehmanniae Praeparata, Cornus officinalis, Rhizoma } \\
\text { alismatis, Chinese yam, Epimedium, Paeonia suffruticosa, } \\
\text { Tuckahoe, Barbary wolfberry fruit, Semen Cuscutae, herba }\end{array}$ & Granule & $\begin{array}{c}\text { SFDA approval } \\
\text { number: Z20050537 }\end{array}$ \\
\hline
\end{tabular}

Cui 2017 [31] Chaihu Jiangu decoction

Cui 2018 [30]

Dou 2015 [32]

Danzi Kangxi electuary

Fu 2018 [33]

Guo 2019 [34]

He 2019 [35]

Hong 2013 [36]

Qufeng Jiangu

Hu 2012 [37]

Huang 2015 [38]

Jiang 2009 [39]
Duhuo Jisheng decoction decoction

Shuanggu Sanzi capsule

uhuo Jisheng
decoction (n)

Bushen Zhuangu decoction

Bushen Guangjie Huoluo Pulvis

Bushen Huoxue decoction

$$
\text { Cistanche, Concha ostreae }
$$

Radix Bupleuri, Scutellaria baicalensis, Rhizoma Pinellinae Praeparata, Codonopsis pilosula, Cassia twig, Radix Paeoniae Alba, Rhizoma Chuanxiong, Achyranthes root, Arisaema cum bile, liquorice

Radix Rehmanniae Praeparata 15 g, Epimedium 15 g, Semen Cuscutae $15 \mathrm{~g}$, Angelica sinensis $10 \mathrm{~g}$, Salviae miltiorrhizae $10 \mathrm{~g}$, Achyranthes root $10 \mathrm{~g}$, Eucommia ulmoides $10 \mathrm{~g}$, Radix Dipsaci 10 g, Radix Codonopsis $15 \mathrm{~g}$, Rhizoma Atractylodis macrocephalae $15 \mathrm{~g}$, liquorice $6 \mathrm{~g}$

Human placenta, Salviae miltiorrhizae, Radix Rehmanniae Praeparata, Achyranthes root, Fructus psoraleae, Morinda officinalis, parasitic Loranthus, woodlouse, Radix Angelicae Pubescentis, Radix Paeoniae Alba

Radix Angelicae Pubescentis $15 \mathrm{~g}$, parasitic Loranthus $15 \mathrm{~g}$, glossy privet fruit $15 \mathrm{~g}$, Semen Cuscutae $15 \mathrm{~g}$, Angelica sinensis $15 \mathrm{~g}$, Eucommia ulmoides $15 \mathrm{~g}$, Barbary wolfberry fruit $15 \mathrm{~g}$, Radix Sileris $15 \mathrm{~g}$, Semen Psoraleae $15 \mathrm{~g}$, Achyranthes root $15 \mathrm{~g}$, peach seed $10 \mathrm{~g}$, flowers carthami $10 \mathrm{~g}$, Rhizoma Chuanxiong $10 \mathrm{~g}$, herba Lycopi $10 \mathrm{~g}$, liquorice $6 \mathrm{~g}$, Asarum $3 \mathrm{~g}$

Radix Angelicae Pubescentis $15 \mathrm{~g}$, parasitic Loranthus $15 \mathrm{~g}$, glossy privet fruit $15 \mathrm{~g}$, Semen Cuscutae $15 \mathrm{~g}$, Angelica sinensis $15 \mathrm{~g}$,

Eucommia ulmoides $15 \mathrm{~g}$, Barbary wolfberry fruit $15 \mathrm{~g}$, Radix

Sileris $15 \mathrm{~g}$, Semen Psoraleae $15 \mathrm{~g}$, Achyranthes root $15 \mathrm{~g}$, peach seed $10 \mathrm{~g}$, flowers carthami $10 \mathrm{~g}$, Rhizoma Chuanxiong $10 \mathrm{~g}$, herba Lycopi $10 \mathrm{~g}$, liquorice $6 \mathrm{~g}$, Asarum $3 \mathrm{~g}$

Woodlouse, Rhizoma Drynariae, Pyritum, Astragalus, Angelica sinensis, Resina Draconis, Rheum officinale, olibanum, myrrh,

Rhizoma Cibotii, Semen Allii Tuberosi, muskmelon seed, cucumber seed

Rhizoma seu Radix Notopterygii $30 \mathrm{~g}$, Caulis Spatholobi $30 \mathrm{~g}$, Radix Cynanchi Panicullati 30 g, Rhizoma Chuanxiong 15 g,

Rhizoma Curcumae 15 g, Radix Curcumae 15 g, Rhizoma

Drynariae $15 \mathrm{~g}$, obscured homalomena rhizome $15 \mathrm{~g}$, Berba Aristolochiae Mollissimae 15 g, liquorice 6 g, Pericarpium Citri Reticulatae $6 \mathrm{~g}$

Radix Rehmanniae Praeparata 15 g, Epimedium 15 g, Semen Cuscutae $15 \mathrm{~g}$, Angelica sinensis $10 \mathrm{~g}$, Salviae miltiorrhizae $10 \mathrm{~g}$, Achyranthes root $10 \mathrm{~g}$, Eucommia ulmoides $10 \mathrm{~g}$, Radix Dipsaci $10 \mathrm{~g}$, Radix Codonopsis $15 \mathrm{~g}$, Rhizoma Atractylodis macrocephalae $15 \mathrm{~g}$, liquorice $6 \mathrm{~g}$

Semen Cuscutae $10 \mathrm{~g}$, Rhizoma Atractylodis macrocephalae $20 \mathrm{~g}$, Morinda officinalis $20 \mathrm{~g}$, Notoginseng Radix $15 \mathrm{~g}$, Radix Curcumae Longae $15 \mathrm{~g}$, Achyranthes root $30 \mathrm{~g}$, Radix Arnebiae seu Lithospermi $15 \mathrm{~g}$, Rhizoma Alismatis $20 \mathrm{~g}$, desert Cistanche $20 \mathrm{~g}$, Radix Sileris 20 g, liquorice 5 g, Rhizoma Chuanxiong 20 g, Radix Arnebiae seu Lithospermi $15 \mathrm{~g}$, cowherb seed $15 \mathrm{~g}$

Radix Rehmanniae Praeparata 15 g, Epimedium 15 g, Semen Cuscutae $15 \mathrm{~g}$, Angelica sinensis $10 \mathrm{~g}$, Salviae miltiorrhizae $10 \mathrm{~g}$, Achyranthes root $10 \mathrm{~g}$, Eucommia ulmoides $10 \mathrm{~g}$, Radix Dipsaci

Decoction Hospital preparation

Decoction Hospital preparation

Decoction

Capsule

Decoction

Decoction Hospital preparation

Pulvis Hospital preparation

Decoction Hospital preparation
SFDA approval number: B20020788 
TABle 3: Continued.

\begin{tabular}{|c|c|c|c|c|}
\hline Study & $\begin{array}{l}\text { Prescription } \\
\text { name }\end{array}$ & Ingredients of herb prescription & Preparations & Quality control \\
\hline Li 2018 [40] & $\begin{array}{l}\text { Duhuo Jisheng } \\
\text { decoction }\end{array}$ & $\begin{array}{c}10 \mathrm{~g} \text {, Radix Codonopsis } 15 \mathrm{~g} \text {, Rhizoma Atractylodis macrocephalae } \\
15 \mathrm{~g} \text {, liquorice } 6 \mathrm{~g} \\
\text { Radix Angelicae Pubescentis } 15 \mathrm{~g} \text {, parasitic Loranthus } 15 \mathrm{~g} \text {, glossy } \\
\text { privet fruit } 15 \mathrm{~g} \text {, Semen Cuscutae } 15 \mathrm{~g} \text {, Angelica sinensis } 15 \mathrm{~g}, \\
\text { Eucommia ulmoides } 15 \mathrm{~g} \text {, Barbary wolfberry fruit } 15 \mathrm{~g} \text {, Radix } \\
\text { Sileris } 15 \mathrm{~g} \text {, Semen Psoraleae } 15 \mathrm{~g} \text {, Achyranthes root } 15 \mathrm{~g} \text {, peach } \\
\text { seed } 10 \mathrm{~g} \text {, flowers carthami } 10 \mathrm{~g} \text {, Rhizoma Chuanxiong } 10 \mathrm{~g} \text {, } \\
\text { herba Lycopi } 10 \mathrm{~g} \text {, liquorice } 6 \mathrm{~g} \text {, Asarum } 3 \mathrm{~g}\end{array}$ & Decoction & Hospital preparation \\
\hline Liu 2011 [43] & $\begin{array}{l}\text { Zhengqing } \\
\text { Fengtongning } \\
\text { tablet }\end{array}$ & Sinomenine $60 \mathrm{mg}$ & Tablet & $\begin{array}{l}\text { SFDA approval } \\
\text { number: Z20010174 }\end{array}$ \\
\hline Liu 2016 [41] & $\begin{array}{l}\text { Jingu Tongning } \\
\text { capsule }\end{array}$ & $\begin{array}{c}\text { Rheum officinale, woodlouse, olibanum, myrrh, Angelica sinensis, } \\
\text { flowers carthami, Radix Paeoniae Alba, Fructus forsythiae, } \\
\text { Fructus gardeniae, borneol }\end{array}$ & Capsule & $\begin{array}{l}\text { Henan FDA } \\
\text { approval number: Z } \\
204090031\end{array}$ \\
\hline Liu 2017 [42] & Shujin decoction & $\begin{array}{c}\text { Astragalus } 15 \mathrm{~g} \text {, Epimedium } 15 \mathrm{~g} \text {, cornu Cervi degelatinatum } 15 \mathrm{~g} \text {, } \\
\text { Rhizoma Chuanxiong } 15 \mathrm{~g} \text {, Radix Rehmanniae Praeparata } 15 \mathrm{~g} \text {, } \\
\text { Radix Angelicae Pubescentis } 15 \mathrm{~g}, \text { Radix Aconiti Lateralis } \\
\text { Preparata } 10 \mathrm{~g}, \text { Lycopodium clavatum } 10 \mathrm{~g} \text {, Rhizoma seu Radix } \\
\text { Notopterygii } 10 \mathrm{~g}, \text { Barbary wolfberry fruit } 10 \mathrm{~g} \text {, Angelica sinensis } \\
10 \mathrm{~g} \text {, Radix Sileris } 10 \mathrm{~g}, \text { Kadsura pepper stem } 8 \mathrm{~g} \text {, Radix Paeoniae } \\
\text { Alba } 30 \mathrm{~g} \text {, Fructus cnidii } 12 \mathrm{~g} \text {, Caulis Spatholobi } 20 \mathrm{~g} \text {, Elecampane } \\
8 \mathrm{~g} \text {, liquorice } 8 \mathrm{~g}\end{array}$ & Decoction & Hospital preparation \\
\hline Lu 2016 [44] & $\begin{array}{l}\text { Lujiao } \\
\text { Zhuanggu } \\
\text { capsule }\end{array}$ & $\begin{array}{l}\text { Cornu Cervi Degelatinatum, Fructus psoraleae, Astragalus, } \\
\text { wolfberry, Rhizoma Chuanxiong, Rheum officinale, Radix Dipsaci }\end{array}$ & Capsule & $\begin{array}{l}\text { Guizhou EDA } \\
\text { approval number: } \\
\text { Z20120003 }\end{array}$ \\
\hline Luo 2019 [45] & $\begin{array}{l}\text { Zhengqing } \\
\text { Fengtongning } \\
\text { tablet }\end{array}$ & Sinomenine $60 \mathrm{mg}$ & Tablet & $\begin{array}{l}\text { SFDA approval } \\
\text { number: Z20010174 }\end{array}$ \\
\hline Ma 2009 [48] & $\begin{array}{l}\text { Huoxue } \\
\text { Tongluo } \\
\text { decoction }\end{array}$ & $\begin{array}{c}\text { Peach seed } 10 \mathrm{~g} \text {, flowers carthami } 12 \mathrm{~g} \text {, Rhizoma Chuanxiong } \\
10 \mathrm{~g} \text {, Angelica sinensis } 12 \mathrm{~g} \text {, root of common peony } 10 \mathrm{~g}, \\
\text { Achyranthes root } 12 \mathrm{~g} \text {, parasitic Loranthus } 15 \mathrm{~g} \text {, Eucommia } \\
\text { ulmoides } 12 \mathrm{~g} \text {, Radix Clematidis } 12 \mathrm{~g}\end{array}$ & Decoction & Hospital preparation \\
\hline Ma 2018 [46] & $\begin{array}{l}\text { Duhuo Jisheng } \\
\text { decoction }\end{array}$ & $\begin{array}{l}\text { Radix angelicae pubescentis } 15 \mathrm{~g} \text {, Parasitic loranthus } 15 \mathrm{~g} \text {, glossy } \\
\text { privet fruit } 15 \mathrm{~g} \text {, Semen cuscutae } 15 \mathrm{~g} \text {, Angelica sinensis } 15 \mathrm{~g} \text {, } \\
\text { Eucommia ulmoides } 15 \mathrm{~g} \text {, Barbary Wolfberry fruit } 15 \mathrm{~g} \text {, Radix } \\
\text { Sileris } 15 \mathrm{~g} \text {, semen psoraleae } 15 \mathrm{~g} \text {, Achyranthes root } 15 \mathrm{~g} \text {, peach } \\
\text { seed } 10 \mathrm{~g} \text {, flowers carthami } 10 \mathrm{~g} \text {, Rhizoma Chuanxiong } 10 \mathrm{~g} \text {, } \\
\text { Herba Lycopi } 10 \mathrm{~g} \text {, liquorice } 6 \mathrm{~g} \text {, Asarum } 3 \mathrm{~g}\end{array}$ & Decoction & Hospital preparation \\
\hline Ma 2019 [47] & $\begin{array}{l}\text { Bushen Yiqi } \\
\text { Huayu Jiedu } \\
\text { decoction }\end{array}$ & $\begin{array}{c}\text { Astragalus } 15 \mathrm{~g} \text {, Fructus psoraleae } 15 \mathrm{~g} \text {, herba Cistanche } 15 \mathrm{~g}, \\
\text { Radix Codonopsis } 12 \mathrm{~g} \text {, Rhizoma Chuanxiong } 9 \mathrm{~g} \text {, Salviae } \\
\text { miltiorrhizae } 9 \mathrm{~g} \text {, synthetic musk } 0.03 \mathrm{~g}\end{array}$ & Decoction & Hospital preparation \\
\hline Mo 2018 [49] & $\begin{array}{l}\text { Kangguzhi } \\
\text { Zengsheng } \\
\text { capsule }\end{array}$ & $\begin{array}{c}\text { Radix Rehmanniae Praeparata, desert Cistanche, Rhizoma } \\
\text { Cibotii, glossy privet fruit, Epimedium, Caulis Spatholobi, Radish } \\
\text { seed, Rhizoma Drynariae, Achyranthes root }\end{array}$ & Capsule & $\begin{array}{c}\text { SFDA approval } \\
\text { number: Z10980006 }\end{array}$ \\
\hline Pan 2017 [22] & Longbie capsule & $\begin{array}{c}\text { Morinda officinalis, Rhizoma Curculiginis, Semen Cuscutae, } \\
\text { Scorpio, centipede, Agkistrodon, woodlouse, Salviae } \\
\text { miltiorrhizae, Radix Aconiti Preparata }\end{array}$ & Capsule & $\begin{array}{l}\text { Guangdong FDA } \\
\text { approval number: } \\
\text { Z20071030 }\end{array}$ \\
\hline Qian 2019 [51] & $\begin{array}{l}\text { Yishen Quyu } \\
\text { decoction }\end{array}$ & $\begin{array}{l}\text { Rhizoma Chuanxiong } 18 \mathrm{~g} \text {, Chinese angelica } 18 \mathrm{~g} \text {, root of } \\
\text { Achyranthes bidentata } 18 \mathrm{~g} \text {, Rhizoma Drynariae } 18 \mathrm{~g} \text {, Rhizoma } \\
\text { Cibotii } 15 \mathrm{~g} \text {, Epimedium } 15 \mathrm{~g} \text {, pubescent angelica root } 12 \mathrm{~g} \text {, } \\
\text { Eucommia ulmoides } 12 \mathrm{~g} \text {, Radix Dipsaci } 12 \mathrm{~g}\end{array}$ & Decoction & Hospital preparation \\
\hline Ren 2016 [53] & $\begin{array}{l}\text { Yishen Quyu } \\
\text { decoction }\end{array}$ & $\begin{array}{l}\text { Rhizoma Chuanxiong } 18 \mathrm{~g} \text {, Angelica sinensis } 18 \mathrm{~g} \text {, Achyranthes } \\
\text { root } 18 \mathrm{~g} \text {, Rhizoma Drynariae } 18 \mathrm{~g} \text {, Rhizoma Cibotii } 15 \mathrm{~g} \text {, } \\
\text { Epimedium } 15 \mathrm{~g} \text {, Radix Angelicae Pubescentis } 12 \mathrm{~g} \text {, Morinda } \\
\text { officinalis } 12 \mathrm{~g} \text {, Eucommia ulmoides } 12 \mathrm{~g} \text {, Radix Dipsaci } 12 \mathrm{~g}\end{array}$ & Decoction & Hospital preparation \\
\hline
\end{tabular}


TABle 3: Continued.

\begin{tabular}{|c|c|c|c|c|}
\hline Study & $\begin{array}{l}\text { Prescription } \\
\text { name }\end{array}$ & Ingredients of herb prescription & Preparations & Quality control \\
\hline Ren 2018 [52] & $\begin{array}{l}\text { Duhuo Jisheng } \\
\text { decoction }\end{array}$ & $\begin{array}{l}\text { Radix Angelicae Pubescentis } 15 \mathrm{~g} \text {, parasitic Loranthus } 15 \mathrm{~g} \text {, glossy } \\
\text { privet fruit } 15 \mathrm{~g} \text {, Semen Cuscutae } 15 \mathrm{~g} \text {, Angelica sinensis } 15 \mathrm{~g}, \\
\text { Eucommia ulmoides } 15 \mathrm{~g} \text {, Barbary wolfberry fruit } 15 \mathrm{~g} \text {, Radix } \\
\text { Sileris } 15 \mathrm{~g} \text {, Semen Psoraleae } 15 \mathrm{~g} \text {, Achyranthes root } 15 \mathrm{~g} \text {, peach } \\
\text { seed } 10 \mathrm{~g} \text {, flowers carthami } 10 \mathrm{~g} \text {, Rhizoma Chuanxiong } 10 \mathrm{~g}, \\
\text { herba Lycopi } 10 \mathrm{~g} \text {, liquorice } 6 \mathrm{~g} \text {, Asarum } 3 \mathrm{~g}\end{array}$ & Decoction & Hospital preparation \\
\hline Rong 2017 [54] & $\begin{array}{l}\text { Duhuo Jisheng } \\
\text { decoction }\end{array}$ & $\begin{array}{c}\text { Radix Angelicae Pubescentis } 15 \mathrm{~g} \text {, parasitic Loranthus } 15 \mathrm{~g} \text {, glossy } \\
\text { privet fruit } 15 \mathrm{~g} \text {, Semen Cuscutae } 15 \mathrm{~g} \text {, Angelica sinensis } 15 \mathrm{~g} \text {, } \\
\text { Eucommia ulmoides } 15 \mathrm{~g} \text {, Barbary wolfberry fruit } 15 \mathrm{~g} \text {, Radix } \\
\text { Sileris } 15 \mathrm{~g} \text {, Semen Psoraleae } 15 \mathrm{~g} \text {, Achyranthes root } 15 \mathrm{~g} \text {, peach } \\
\text { seed } 10 \mathrm{~g} \text {, flowers carthami } 10 \mathrm{~g} \text {, Rhizoma Chuanxiong } 10 \mathrm{~g} \text {, } \\
\text { herba Lycopi } 10 \mathrm{~g} \text {, liquorice } 6 \mathrm{~g} \text {, Asarum } 3 \mathrm{~g}\end{array}$ & Decoction & Hospital preparation \\
\hline Shi 2019 [55] & $\begin{array}{l}\text { Duhuo Jisheng } \\
\text { decoction }\end{array}$ & $\begin{array}{l}\text { Radix Angelicae Pubescentis } 15 \mathrm{~g} \text {, parasitic Loranthus } 15 \mathrm{~g} \text {, glossy } \\
\text { privet fruit } 15 \mathrm{~g} \text {, Semen Cuscutae } 15 \mathrm{~g} \text {, Angelica sinensis } 15 \mathrm{~g} \text {, } \\
\text { Eucommia ulmoides } 15 \mathrm{~g} \text {, Barbary wolfberry fruit } 15 \mathrm{~g} \text {, Radix } \\
\text { Sileris } 15 \mathrm{~g} \text {, Semen Psoraleae } 15 \mathrm{~g} \text {, Achyranthes root } 15 \mathrm{~g} \text {, peach } \\
\text { seed } 10 \mathrm{~g} \text {, flowers carthami } 10 \mathrm{~g} \text {, Rhizoma Chuanxiong } 10 \mathrm{~g} \text {, } \\
\text { herba Lycopi } 10 \mathrm{~g} \text {, liquorice } 6 \mathrm{~g} \text {, Asarum } 3 \mathrm{~g}\end{array}$ & Decoction & Hospital preparation \\
\hline Song 2017 [56] & $\begin{array}{l}\text { Duhuo Jisheng } \\
\text { decoction }\end{array}$ & $\begin{array}{c}\text { Radix Angelicae Pubescentis } 15 \mathrm{~g} \text {, parasitic Loranthus } 15 \mathrm{~g} \text {, glossy } \\
\text { privet fruit } 15 \mathrm{~g} \text {, Semen Cuscutae } 15 \mathrm{~g} \text {, Angelica sinensis } 15 \mathrm{~g} \text {, } \\
\text { Eucommia ulmoides } 15 \mathrm{~g} \text {, Barbary wolfberry fruit } 15 \mathrm{~g} \text {, Radix } \\
\text { Sileris } 15 \mathrm{~g} \text {, Semen Psoraleae } 15 \mathrm{~g} \text {, Achyranthes root } 15 \mathrm{~g} \text {, peach } \\
\text { seed } 10 \mathrm{~g} \text {, flowers carthami } 10 \mathrm{~g} \text {, Rhizoma Chuanxiong } 10 \mathrm{~g}, \\
\text { herba Lycopi } 10 \mathrm{~g} \text {, liquorice } 6 \mathrm{~g} \text {, Asarum } 3 \mathrm{~g}\end{array}$ & Decoction & Hospital preparation \\
\hline Sun 2018 [57] & Qinbi decoction & $\begin{array}{c}\text { Chinese starjasmine stem } 12 \mathrm{~g} \text {, Caulis Sinomenii } 12 \mathrm{~g} \text {, } \\
\text { honeysuckle stem } 12 \mathrm{~g} \text {, Semen Coicis } 21 \mathrm{~g} \text {, Tuckahoe } 15 \mathrm{~g} \text {, } \\
\text { Achyranthes root } 12 \mathrm{~g} \text {, parasitic Loranthus } 15 \mathrm{~g} \text {, Radix Stephaniae } \\
\text { Tetrandrae } 9 \mathrm{~g} \text {, Cortex Phellodendri } 9 \mathrm{~g} \text {, root of common peony } \\
9 \mathrm{~g} \text {, Radix Clematidis } 9 \mathrm{~g} \text {, parasitic Loranthus } 9 \mathrm{~g} \text {, Rhizoma } \\
\text { Corydalis } 6 \mathrm{~g} \text {, liquorice } 6 \mathrm{~g}\end{array}$ & Decoction & Hospital preparation \\
\hline Tan 2014 [58] & $\begin{array}{l}\text { Huangqi Biejia } \\
\text { pill }\end{array}$ & $\begin{array}{l}\text { Ginseng, Cortex Cinnamomi, Radix Rehmanniae Recen, Pinellia } \\
\text { ternata, Radix Asteris, Rhizoma Anemarrhenae, Astragalus, } \\
\text { liquorice, Radix Asparagi, Carapax Trionycis, Gentiana } \\
\text { macrophylla, white poria, Cortex Lycii Radicis, Radix Bupleuri }\end{array}$ & Pill & Hospital preparation \\
\hline Tang 2012 [59] & Jiedu Yishen pill & $\begin{array}{c}\text { Radix Cynanchi Panicullati } 120 \mathrm{~g} \text {, Achyranthes root } 80 \mathrm{~g} \text {, } \\
\text { Eucommia ulmoides } 80 \mathrm{~g} \text {, Rhizoma Chuanxiong } 80 \mathrm{~g} \text {, woodlouse } \\
40 \mathrm{~g} \text {, Cortex Phellodendri } 40 \mathrm{~g}\end{array}$ & Pill & Hospital preparation \\
\hline $\begin{array}{l}\text { Wang } 2013 \\
{[60]}\end{array}$ & $\begin{array}{l}\text { Fufang Xiatian } \\
\text { Wu pill }\end{array}$ & $\begin{array}{c}\text { Corydalis amabilis, Radix Aconiti Kusnezoffi Preparata, herba } \\
\text { Siegesbeckiae, Cissus assamica, Caulis Spatholobi, Paederia } \\
\text { scandens, Radix Clematidis, Aristolochia fangchi, Cortex } \\
\text { Acanthopanacis, Rhizoma seu Radix Notopterygii, Gentiana } \\
\text { macrophylla, Agkistrodon, herba ephedra }\end{array}$ & Pill & $\begin{array}{c}\text { SFDA approval } \\
\text { number: Z20003105 }\end{array}$ \\
\hline $\begin{array}{l}\text { Wang } 2019 \\
{[61]}\end{array}$ & $\begin{array}{l}\text { Taoren Xikang } \\
\text { pill }\end{array}$ & $\begin{array}{l}\text { Peach seed, flowers carthami, Angelica sinensis, Radix } \\
\text { Rehmanniae Praeparata, Rhizoma Chuanxiong, Radix Paeoniae } \\
\text { Alba, Radix Angelicae Pubescentis, Radix Sileris, parasitic } \\
\text { Loranthus, Achyranthes root, Asarum, olibanum, myrrh }\end{array}$ & Pill & $\begin{array}{l}\text { Henan FDA } \\
\text { approval number: } \\
\text { Z20120243 }\end{array}$ \\
\hline Wen 2016 [62] & $\begin{array}{l}\text { Duhuo Jisheng } \\
\text { decoction }\end{array}$ & $\begin{array}{l}\text { Radix Angelicae Pubescentis } 15 \mathrm{~g} \text {, parasitic Loranthus } 15 \mathrm{~g} \text {, glossy } \\
\text { privet fruit } 15 \mathrm{~g} \text {, Semen Cuscutae } 15 \mathrm{~g} \text {, Angelica sinensis } 15 \mathrm{~g}, \\
\text { Eucommia ulmoides } 15 \mathrm{~g} \text {, Barbary wolfberry fruit } 15 \mathrm{~g} \text {, Radix } \\
\text { Sileris } 15 \mathrm{~g} \text {, Semen Psoraleae } 15 \mathrm{~g} \text {, Achyranthes root } 15 \mathrm{~g} \text {, peach } \\
\text { seed } 10 \mathrm{~g} \text {, flowers carthami } 10 \mathrm{~g} \text {, Rhizoma Chuanxiong } 10 \mathrm{~g}, \\
\text { herba Lycopi } 10 \mathrm{~g} \text {, liquorice } 6 \mathrm{~g} \text {, Asarum } 3 \mathrm{~g}\end{array}$ & Decoction & Hospital preparation \\
\hline Wu 2012 [65] & $\begin{array}{l}\text { Zhuanggu } \\
\text { Tongbi pill }\end{array}$ & $\begin{array}{l}\text { Radix Rehmanniae Praeparata } 12 \mathrm{~g} \text {, Eucommia ulmoides } 15 \mathrm{~g} \text {, } \\
\text { herba Pyrolae } 30 \mathrm{~g} \text {, pulp of dogwood fruit } 15 \mathrm{~g} \text {, Rhizoma } \\
\text { Drynariae } 15 \mathrm{~g}, \text { Radix Clematidis } 30 \mathrm{~g} \text {, Radix Dipsaci } 15 \mathrm{~g} \text {, } \\
\text { Achyranthes root } 15 \mathrm{~g} \text {, garden balsam stem } 15 \mathrm{~g} \text {, Chinese }\end{array}$ & Pill & Hospital preparation \\
\hline
\end{tabular}


TABle 3: Continued.

\begin{tabular}{|c|c|c|c|c|}
\hline Study & $\begin{array}{l}\text { Prescription } \\
\text { name }\end{array}$ & Ingredients of herb prescription & Preparations & Quality control \\
\hline Wu 2018 [63] & $\begin{array}{c}\text { Bushen Huoxue } \\
\text { decoction }\end{array}$ & $\begin{array}{c}\text { Polyphaga 9 g, Tuckahoe } 12 \mathrm{~g}, \text { Radix Aconiti Preparata } 9 \mathrm{~g}, \\
\text { Fructus psoraleae } 15 \mathrm{~g} \text {, parasitic Loranthus } 15 \mathrm{~g} \\
\text { Radix Rehmanniae Praeparata } 15 \mathrm{~g} \text {, Epimedium } 15 \mathrm{~g} \text {, Semen } \\
\text { Cuscutae } 15 \mathrm{~g} \text {, Angelica sinensis } 10 \mathrm{~g} \text {, Salviae miltiorrhizae } 10 \mathrm{~g} \text {, } \\
\text { Achyranthes root } 10 \mathrm{~g} \text {, Eucommia ulmoides } 10 \mathrm{~g} \text {, Radix Dipsaci } \\
10 \mathrm{~g} \text {, Radix Codonopsis } 15 \mathrm{~g} \text {, Rhizoma Atractylodis macrocephalae } \\
15 \mathrm{~g} \text {, liquorice } 6 \mathrm{~g}\end{array}$ & Decoction & Hospital preparation \\
\hline Wu 2018 [64] & $\begin{array}{l}\text { Sanqi Xuejie } \\
\text { capsule }\end{array}$ & Notoginseng root Radix, Resina Draconis & Capsule & Hospital preparation \\
\hline Xia 2017 [66] & $\begin{array}{l}\text { Jinwu Gutong } \\
\text { capsule }\end{array}$ & $\begin{array}{c}\text { Rhizoma Cibotii, Epimedium, Radix Clematidis, Zaocys } \\
\text { dhumnade, Achyranthes root, Chinese quince, root of kudzu vine, } \\
\text { Radix Curcumae Longae, Fructus psoraleae, Radix } \\
\text { Campanumoeae }\end{array}$ & Capsule & $\begin{array}{c}\text { SFDA approval } \\
\text { number: Z20043621 }\end{array}$ \\
\hline Yang 2016 [67] & $\begin{array}{c}\text { Jiawei Simiao } \\
\text { Pulvis }\end{array}$ & $\begin{array}{c}\text { Cortex Phellodendri } 5 \mathrm{~g} \text {, Rhizoma Atractylodis } 10 \mathrm{~g} \text {, Semen } \\
\text { Coicis } 10 \mathrm{~g} \text {, Achyranthes root } 10 \mathrm{~g} \text {, Radix Stephaniae Tetrandrae } \\
10 \mathrm{~g} \text {, Fructus forsythiae } 10 \mathrm{~g} \text {, Radix Sophorae Flavescentis } 10 \mathrm{~g} \text {, } \\
\text { Chinese quince } 10 \mathrm{~g} \text {, Gentiana macrophylla } 10 \mathrm{~g} \text {, Radix } \\
\text { Rehmanniae Recen } 15 \mathrm{~g} \text {, honeysuckle stem } 15 \mathrm{~g}\end{array}$ & Pulvis & Hospital preparation \\
\hline Yang 2019 [68] & $\begin{array}{l}\text { Duhuo Jisheng } \\
\text { decoction }\end{array}$ & $\begin{array}{l}\text { Radix Angelicae Pubescentis } 15 \mathrm{~g} \text {, parasitic Loranthus } 15 \mathrm{~g} \text {, glossy } \\
\text { privet fruit } 15 \mathrm{~g} \text {, Semen Cuscutae } 15 \mathrm{~g} \text {, Angelica sinensis } 15 \mathrm{~g} \text {, } \\
\text { Eucommia ulmoides } 15 \mathrm{~g} \text {, Barbary wolfberry fruit } 15 \mathrm{~g} \text {, Radix } \\
\text { Sileris } 15 \mathrm{~g} \text {, Semen Psoraleae } 15 \mathrm{~g} \text {, Achyranthes root } 15 \mathrm{~g} \text {, peach } \\
\text { seed } 10 \mathrm{~g} \text {, flowers carthami } 10 \mathrm{~g} \text {, Rhizoma Chuanxiong } 10 \mathrm{~g} \text {, } \\
\text { herba Lycopi } 10 \mathrm{~g} \text {, liquorice } 6 \mathrm{~g} \text {, Asarum } 3 \mathrm{~g}\end{array}$ & Decoction & Hospital preparation \\
\hline Yao 2013 [69] & $\begin{array}{l}\text { Duhuo Jisheng } \\
\text { decoction }\end{array}$ & $\begin{array}{l}\text { Radix Angelicae Pubescentis } 15 \mathrm{~g} \text {, parasitic Loranthus } 15 \mathrm{~g} \text {, glossy } \\
\text { privet fruit } 15 \mathrm{~g} \text {, Semen Cuscutae } 15 \mathrm{~g} \text {, Angelica sinensis } 15 \mathrm{~g} \text {, } \\
\text { Eucommia ulmoides } 15 \mathrm{~g} \text {, Barbary wolfberry fruit } 15 \mathrm{~g} \text {, Radix } \\
\text { Sileris } 15 \mathrm{~g} \text {, Semen Psoraleae } 15 \mathrm{~g} \text {, Achyranthes root } 15 \mathrm{~g} \text {, peach } \\
\text { seed } 10 \mathrm{~g} \text {, flowers carthami } 10 \mathrm{~g} \text {, Rhizoma Chuanxiong } 10 \mathrm{~g} \text {, } \\
\text { herba Lycopi } 10 \mathrm{~g} \text {, liquorice } 6 \mathrm{~g} \text {, Asarum } 3 \mathrm{~g}\end{array}$ & Decoction & Hospital preparation \\
\hline Yi 2017 [70] & $\begin{array}{c}\text { Bushen Huoxue } \\
\text { decoction }\end{array}$ & $\begin{array}{c}\text { Radix Rehmanniae Praeparata } 15 \mathrm{~g} \text {, Epimedium } 15 \mathrm{~g} \text {, Semen } \\
\text { Cuscutae } 15 \mathrm{~g} \text {, Angelica sinensis } 10 \mathrm{~g} \text {, Salviae miltiorrhizae } 10 \mathrm{~g} \text {, } \\
\text { Achyranthes root } 10 \mathrm{~g} \text {, Eucommia ulmoides } 10 \mathrm{~g} \text {, Radix Dipsaci } \\
10 \mathrm{~g} \text {, Radix Codonopsis } 15 \mathrm{~g} \text {, Rhizoma Atractylodis macrocephalae } \\
15 \mathrm{~g} \text {, liquorice } 6 \mathrm{~g}\end{array}$ & Decoction & Hospital preparation \\
\hline Yi 2017 [71] & $\begin{array}{l}\text { Qiangjin } \\
\text { Zhuanggu } \\
\text { decoction }\end{array}$ & $\begin{array}{l}\text { Semen Cuscutae } 15 \text { g, Rhizoma Curculiginis } 20 \text { g, Morinda } \\
\text { officinalis } 10 \text { g, Radix Rehmanniae Praeparata } 15 \text { g, parasitic } \\
\text { Loranthus } 10 \text { g, Radix Aconiti Preparata } 9 \text { g, Scorpio } 4 \text { g, } \\
\text { centipede } 4 \text { g, Radix Clematidis } 15 \text { g, Lycopodium clavatum } 15 \text { g, } \\
\text { Caulis Spatholobi } 15 \text { g, Rhizoma Chuanxiong } 15 \text { g, Ramulus mori } \\
10 \text { g, Radix Sileris } 10 \text { g, Tribulus terrestris } 15 \text { g, liquorice } 10 \mathrm{~g}\end{array}$ & Decoction & Hospital preparation \\
\hline Yu $2010[72]$ & $\begin{array}{l}\text { Duhuo Jisheng } \\
\text { decoction }\end{array}$ & $\begin{array}{c}\text { Radix Angelicae Pubescentis } 15 \mathrm{~g} \text {, parasitic Loranthus } 15 \mathrm{~g} \text {, glossy } \\
\text { privet fruit } 15 \mathrm{~g} \text {, Semen Cuscutae } 15 \mathrm{~g} \text {, Angelica sinensis } 15 \mathrm{~g}, \\
\text { Eucommia ulmoides } 15 \mathrm{~g} \text {, Barbary wolfberry fruit } 15 \mathrm{~g} \text {, Radix } \\
\text { Sileris } 15 \mathrm{~g} \text {, Semen Psoraleae } 15 \mathrm{~g} \text {, Achyranthes root } 15 \mathrm{~g} \text {, peach } \\
\text { seed } 10 \mathrm{~g} \text {, flowers carthami } 10 \mathrm{~g} \text {, Rhizoma Chuanxiong } 10 \mathrm{~g} \text {, } \\
\text { herba Lycopi } 10 \mathrm{~g} \text {, liquorice } 6 \mathrm{~g} \text {, Asarum } 3 \mathrm{~g}\end{array}$ & Decoction & Hospital preparation \\
\hline Yuan 2017 [73] & $\begin{array}{c}\text { Bushen Huoxue } \\
\text { decoction }\end{array}$ & $\begin{array}{c}\text { Radix Rehmanniae Praeparata } 15 \mathrm{~g} \text {, Epimedium } 15 \mathrm{~g} \text {, Semen } \\
\text { Cuscutae } 15 \mathrm{~g} \text {, Angelica sinensis } 10 \mathrm{~g} \text {, Salviae miltiorrhizae } 10 \mathrm{~g} \text {, } \\
\text { Achyranthes root } 10 \mathrm{~g} \text {, Eucommia ulmoides } 10 \mathrm{~g} \text {, Radix Dipsaci } \\
10 \mathrm{~g} \text {, Radix Codonopsis } 15 \mathrm{~g} \text {, Rhizoma Atractylodis macrocephalae } \\
15 \mathrm{~g} \text {, liquorice } 6 \mathrm{~g}\end{array}$ & Decoction & Hospital preparation \\
\hline $\begin{array}{l}\text { Zhang } 2016 \\
{[74]}\end{array}$ & $\begin{array}{l}\text { Jinwu Gutong } \\
\text { capsule }\end{array}$ & $\begin{array}{c}\text { Rhizoma Cibotii, Epimedium, Radix Clematidis, Zaocys } \\
\text { dhumnade, Achyranthes root, Chinese quince, root of kudzu vine, } \\
\text { Radix Curcumae Longae, Fructus psoraleae, Radix } \\
\text { Campanumoeae }\end{array}$ & Capsule & $\begin{array}{c}\text { SFDA approval } \\
\text { number: Z20043621 }\end{array}$ \\
\hline
\end{tabular}


TABLE 3: Continued.

\begin{tabular}{|c|c|c|c|c|}
\hline Study & $\begin{array}{l}\text { Prescription } \\
\text { name }\end{array}$ & Ingredients of herb prescription & Preparations & Quality control \\
\hline $\begin{array}{l}\text { Zhang } 2017 \\
\text { [77] }\end{array}$ & $\begin{array}{l}\text { Fugui Gutong } \\
\text { capsule }\end{array}$ & $\begin{array}{c}\text { Radix Aconiti Lateralis Preparata, Radix Aconiti Preparata, } \\
\text { Cortex Cinnamomi, Codonopsis pilosula, Angelica sinensis, Radix } \\
\text { Paeoniae Alba, Epimedium, olibanum }\end{array}$ & Capsule & $\begin{array}{c}\text { SFDA approval } \\
\text { number: Z19990026 }\end{array}$ \\
\hline $\begin{array}{l}\text { Zhang } 2018 \\
{[76]}\end{array}$ & Qianggu capsule & Rhizoma Drynariae $0.25 \mathrm{~g}$ & Capsule & $\begin{array}{c}\text { SFDA approval } \\
\text { number: Z20030007 }\end{array}$ \\
\hline $\begin{array}{l}\text { Zhang } 2019 \\
\text { [75] }\end{array}$ & $\begin{array}{l}\text { Duhuo Jisheng } \\
\text { decoction }\end{array}$ & $\begin{array}{l}\text { Radix Angelicae Pubescentis } 15 \mathrm{~g} \text {, parasitic Loranthus } 15 \mathrm{~g} \text {, glossy } \\
\text { privet fruit } 15 \mathrm{~g} \text {, Semen Cuscutae } 15 \mathrm{~g} \text {, Angelica sinensis } 15 \mathrm{~g} \text {, } \\
\text { Eucommia ulmoides } 15 \mathrm{~g} \text {, Barbary wolfberry fruit } 15 \mathrm{~g} \text {, Radix } \\
\text { Sileris } 15 \mathrm{~g} \text {, Semen Psoraleae } 15 \mathrm{~g} \text {, Achyranthes root } 15 \mathrm{~g} \text {, peach } \\
\text { seed } 10 \mathrm{~g} \text {, flowers carthami } 10 \mathrm{~g} \text {, Rhizoma Chuanxiong } 10 \mathrm{~g} \text {, } \\
\text { herba Lycopi } 10 \mathrm{~g} \text {, liquorice } 6 \mathrm{~g} \text {, Asarum } 3 \mathrm{~g}\end{array}$ & Decoction & Hospital preparation \\
\hline $\begin{array}{l}\text { Zheng } 2014 \\
\text { [78] }\end{array}$ & $\begin{array}{l}\text { Zhengqing } \\
\text { Fengtongning } \\
\text { tablet }\end{array}$ & Sinomenine $60 \mathrm{mg}$ & Tablet & $\begin{array}{c}\text { SFDA approval } \\
\text { number: Z20010174 }\end{array}$ \\
\hline $\begin{array}{l}\text { Zheng } 2019 \\
\text { [79] }\end{array}$ & $\begin{array}{c}\text { Bushen Huoxue } \\
\text { decoction }\end{array}$ & $\begin{array}{c}\text { Radix Rehmanniae Praeparata } 15 \mathrm{~g} \text {, Epimedium } 15 \mathrm{~g} \text {, Semen } \\
\text { Cuscutae } 15 \mathrm{~g} \text {, Angelica sinensis } 10 \mathrm{~g} \text {, Salviae miltiorrhizae } 10 \mathrm{~g}, \\
\text { Achyranthes root } 10 \mathrm{~g} \text {, Eucommia ulmoides } 10 \mathrm{~g} \text {, Radix Dipsaci } \\
10 \mathrm{~g} \text {, Radix Codonopsis } 15 \mathrm{~g} \text {, Rhizoma Atractylodis macrocephalae } \\
15 \mathrm{~g} \text {, liquorice } 6 \mathrm{~g}\end{array}$ & Decoction & Hospital preparation \\
\hline $\begin{array}{l}\text { Zhong } 2017 \\
{[80]}\end{array}$ & $\begin{array}{c}\text { Shentong Zhuyu } \\
\text { decoction }\end{array}$ & $\begin{array}{c}\text { Angelica sinensis } 15 \mathrm{~g} \text {, Rhizoma Chuanxiong } 15 \mathrm{~g} \text {, Achyranthes } \\
\text { root } 15 \mathrm{~g} \text {, peach seed } 12 \mathrm{~g} \text {, flowers carthami } 12 \mathrm{~g} \text {, Gentiana } \\
\text { macrophylla } 12 \mathrm{~g} \text {, Rhizoma seu Radix Notopterygii } 12 \mathrm{~g} \text {, Rhizoma } \\
\text { Cyperi } 9 \mathrm{~g} \text {, myrrh 9g, earthworm } 9 \mathrm{~g} \text {, Trogopterus Dung } 6 \mathrm{~g} \text {, } \\
\text { liquorice } 6 \mathrm{~g}\end{array}$ & Decoction & Hospital preparation \\
\hline Zhou 2012 [81] & $\begin{array}{l}\text { Shufu Jiangu } \\
\text { decoction }\end{array}$ & $\begin{array}{l}\text { Radix Aconiti Lateralis Preparata } 10 \mathrm{~g} \text {, Radix Rehmanniae } \\
\text { Praeparata } 20 \mathrm{~g} \text {, Rhizoma Drynariae } 10 \mathrm{~g} \text {, Radix Dipsaci } 15 \mathrm{~g} \text {, } \\
\text { Radix Angelicae Pubescentis } 10 \mathrm{~g} \text {, Achyranthes root } 15 \mathrm{~g} \text {, Radix } \\
\text { Clematidis } 10 \mathrm{~g} \text {, Salviae miltiorrhizae } 15 \mathrm{~g} \text {, pangolin scales } 10 \mathrm{~g} \text {, } \\
\text { Scorpio } 3 \mathrm{~g} \text {, Ramulus mori } 15 \mathrm{~g} \text {, liquorice } 6 \mathrm{~g}\end{array}$ & Decoction & Hospital preparation \\
\hline Zhu 2013 [82] & $\begin{array}{l}\text { Zhengqing } \\
\text { Fengtongning } \\
\text { tablet }\end{array}$ & Sinomenine $60 \mathrm{mg}$ & Tablet & $\begin{array}{c}\text { SFDA approval } \\
\text { number: Z20010174 }\end{array}$ \\
\hline Zhuo 2019 [83] & $\begin{array}{l}\text { Rendong Bixie } \\
\text { decoction }\end{array}$ & $\begin{array}{l}\text { Honeysuckle stem } 30 \mathrm{~g} \text {, yam rhizome } 15 \mathrm{~g} \text {, Radix Clematidis } 12 \mathrm{~g} \text {, } \\
\text { bark of Himalayan coralbean } 15 \mathrm{~g} \text {, root of common peony } 12 \mathrm{~g} \text {, } \\
\text { Radix Gentianae Macrophyllae } 15 \mathrm{~g} \text {, herba Siegesbeckiae } 15 \mathrm{~g} \text {, } \\
\text { loofah sponge } 20 \mathrm{~g} \text {, Ramulus mori } 30 \mathrm{~g}\end{array}$ & Decoction & Hospital preparation \\
\hline
\end{tabular}

SFDA: State Food and Drug Administration; FDA: Food and Drug Administration.

The pooled results indicated that CHM monotherapy significantly reduced VAS when compared to NSAIDS alone $(\mathrm{SMD}=-0.803 ; 95 \% \mathrm{CI}=-1.158$ to $-0.449 ; P \leq 0.001$; heterogeneity $\chi^{2}=53.68, \quad \mathrm{df}=9, \quad I^{2}=83.2 \%, \quad P \leq 0.001$, Figure 2(b)). We identified five studies that compared CHM plus Glu to Glu alone in terms of the VAS [33, 45, 48, 72, 80]. CHM plus Glu significantly decreased the VAS in comparison to Glu alone (SMD $=-1.204 ; 95 \% \mathrm{CI}=-1.593$ to $0.815 ; P=0.001$; heterogeneity $\chi^{2}=17.89$, df $=4, I^{2}=77.6 \%$ , $P=0.001$, Figure 2(c)). Eight studies compared CHM and Glu on the basis of their VAS scores [32, 35, 36, 43, 51, 58, $66,84]$. The pooled results indicated that CHM monotherapy significantly decreased VAS compared with Glu alone $(\mathrm{SMD}=-1.533 ; 95 \% \mathrm{CI}=-1.688$ to $-1.076 ; P \leq 0.001$; het erogeneity $\chi^{2}=123.94, \quad \mathrm{df}=7, \quad I^{2}=94.4 \%, \quad P \leq 0.001$, Figure 2(d)). Metaregression was used to investigate the sources of heterogeneity in the findings. To ascertain the potential sources of interstudy heterogeneity, we conducted a metaregression analysis on the year of publication, course of treatment, and sample size (Figure 3). In general, the year of publication $\left(\beta=-0.103 ; P=0.120 ; R^{2}=5.20 \%\right)$, the duration of treatment $\left(\beta=0.037 ; P=0.231 ; R^{2}=1.33 \%\right)$, and the sample size $\left(\beta=-0.011 ; P=0.100 ; R^{2}=6.37 \%\right)$, were not significant sources of heterogeneity for the VAS.

3.3.2. WOMAC. Seven studies compared CHM plus NSAIDS to NSAIDS alone in terms of the WOMAC score $[38,44,46,68,75,79,87]$. The pooled data revealed that CHM plus NSAIDS was significantly more effective at reducing WOMAC than NSAIDS alone $(\mathrm{SMD}=-2.131 ; 95$ $\% \mathrm{CI}=-3.082$ to $-1.180 ; \quad P \leq 0.001 ; \quad$ heterogeneity $\chi^{2}=$ 150.92, df $=6, I^{2}=96.0 \%, P \leq 0.001$, Figure $\left.4(\mathrm{a})\right)$. There were three studies comparing CHM and NSAIDS in terms of the WOMAC $[61,73,83]$. The pooled data showed that 


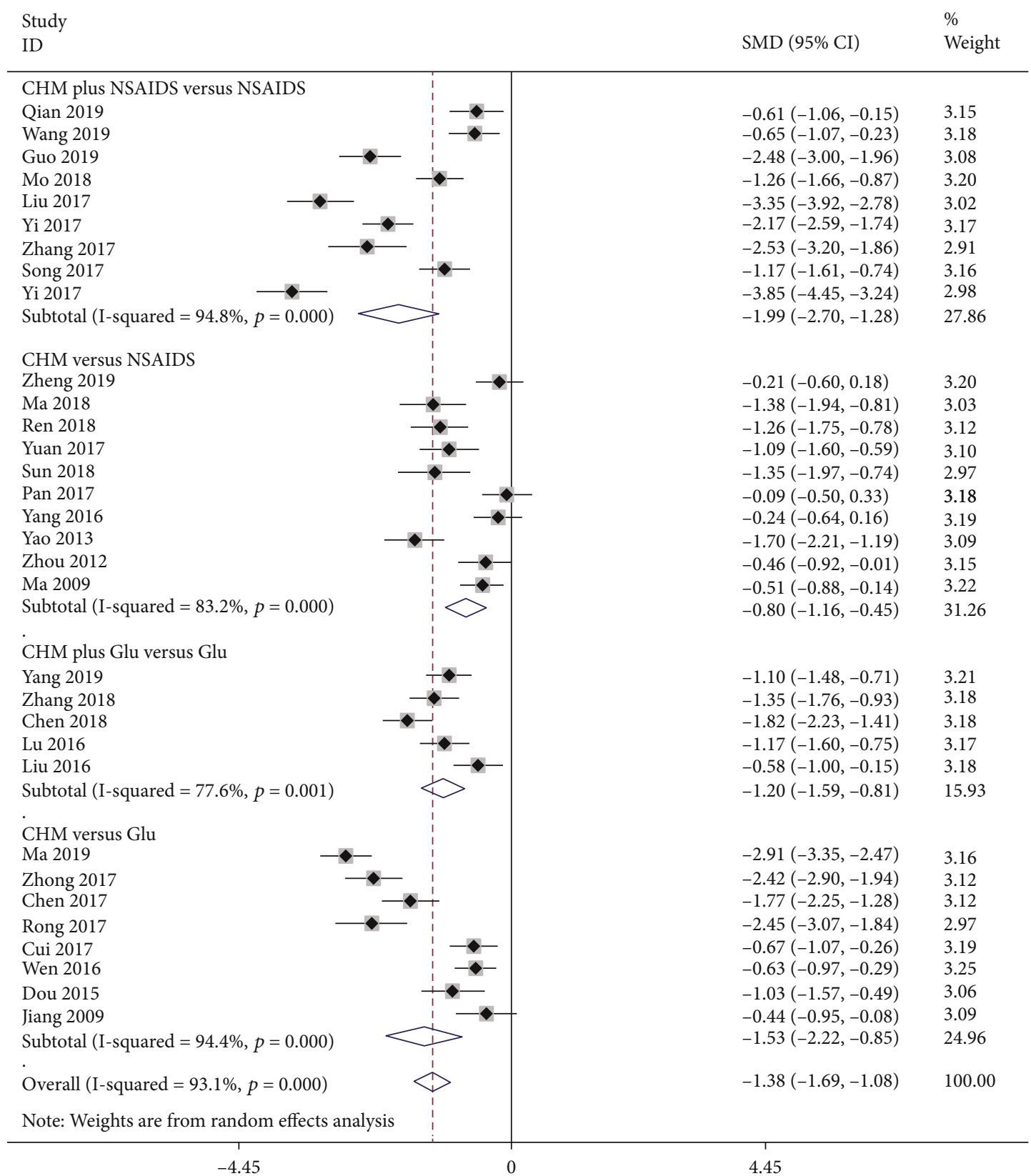

Figure 2: Forest plot of VAS: (a) CHM plus NSAIDS versus NSAIDS. (b) CHM versus NSAIDS. (c) CHM plus Glu versus Glu. (d) CHM versus Glu.

CHM monotherapy significantly decreased WOMAC when compared to NSAIDs alone $(\mathrm{SMD}=-0.672 ; 95 \% \mathrm{CI}=-$ 1.226 to $-0.119 ; P=0.017$; heterogeneity $\chi^{2}=8.19$, $\mathrm{df}=2$, $I^{2}=75.6 \%, P=0.017$, Figure $\left.4(\mathrm{~b})\right)$. Six studies compared CHM plus Glu with Glu alone in terms of WOMAC [33, $40,47,49,59,72]$. The findings indicated that CHM plus Glu significantly decreased WOMAC score when compared to $\mathrm{Glu}$ alone $(\mathrm{SMD}=-1.315 ; 95 \% \mathrm{CI}=-2.162$ to $-0.468 ; P$ $=0.002$; heterogeneity $\chi^{2}=112.54, \mathrm{df}=5, I^{2}=95.6 \%, P \leq$ 0.001 , Figure $4(\mathrm{c}))$. Six studies examined the efficacy of $\mathrm{CHM}$ and Glu in terms of WOMAC. The pooled data indicated that CHM monotherapy was significantly more effec- tive than Glu in reducing WOMAC $(\mathrm{SMD}=-1.095$; $95 \% \mathrm{CI}=-1.607$ to $-0.583 ; P \leq 0.001 ;$ heterogeneity $\chi^{2}=$ $36.84, \mathrm{df}=5, I^{2}=86.4 \%, P \leq 0.001$, Figure $\left.4(\mathrm{~d})\right)$. We used metaregression to determine the sources of heterogeneity in the findings. We also conducted metaregression to examine the year of publication, duration of treatment, and the sample size to ascertain the potential reasons of interstudy heterogeneity (Figure 5). Altogether, the year of publication $\left(\beta=-0.112 ; P=0.171 ; R^{2}=4.83 \%\right)$, duration of treatment $\left(\beta=0.007 ; \quad P=0.861 ; R^{2}=5.18 \%\right)$, and the sample size $\left(\beta=-0.012 ; P=0.303 ; R^{2}=0.24 \%\right)$ were not significant predictors of heterogeneity for WOMAC. 


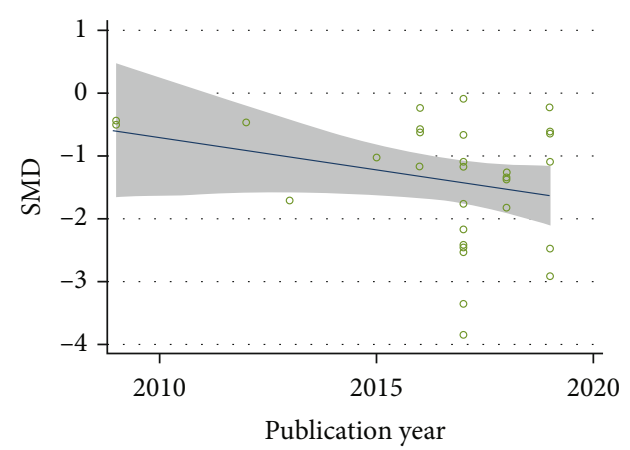

(a)

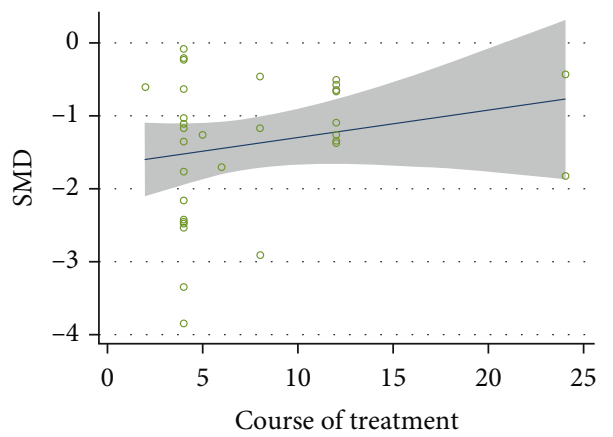

(b)

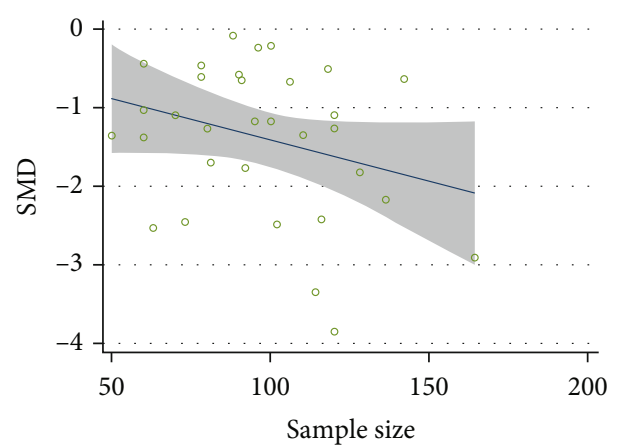

(c)

FIGURE 3: Metaregression analysis of VAS for (a) publication year, (b) course of treatment, (c) and sample size.

3.3.3. Lysholm Score. Five studies compared the Lysholm score between CHM plus NSAIDS and NSAIDS alone [44, 46, 53, 57, 75]. The pooled data indicated that CHM plus NSAIDS was significantly more effective at improving Lysholm score than NSAIDS alone $(\mathrm{SMD}=2.503 ; 95 \% \mathrm{CI}=1.424$ to 3.583 ; $P \leq 0.001$; heterogeneity $\chi^{2}=99.72$, df $=4, I^{2}=96.0 \%, P \leq$ 0.001 , Figure $6(\mathrm{a}))$. There were three studies comparing the Lysholm score of CHM to that of NSAIDS [56, 77, 85]. The pooled data revealed that CHM monotherapy significantly increased the Lysholm score much more than NSAIDS alone $(\mathrm{SMD}=1.071 ; 95 \% \mathrm{CI}=0.459$ to $1.683 ; P=0.001$; heterogeneity $\chi^{2}=9.47, \mathrm{df}=2, I^{2}=78.9 \%, P=0.009$, Figure $\left.6(\mathrm{~b})\right)$.

3.3.4. Lequesne Index. Five studies compared the effect of CHM plus NSAIDS to that of NSAIDS alone on the Lequesne index $[42,55,60,68,81]$. The pooled data revealed that CHM plus NSAIDS treatment significantly decreased the Lequesne index when compared to NSAIDS alone (SMD $=-0.883 ; 95$ $\% \mathrm{CI}=-1.095$ to $-0.672 ; P \leq 0.001$; heterogeneity $\chi^{2}=3.63$, $\mathrm{df}=4, I^{2}=0 \%, P=0.458$, Figure $\left.7(\mathrm{a})\right)$. There was only one study comparing the Lequesne index between CHM and NSAIDS [54]. The results indicated that CHM monotherapy significantly decreased the Lequesne index when compared to NSAIDS alone (SMD $=-0.804 ; 95 \% \mathrm{CI}=-1.239$ to 0.369; $P \leq 0.001$, no heterogeneity, Figure $7(\mathrm{~b}))$. Six studies compared the Lequesne index of CHM plus Glu to Glu alone $[33,41,64,78,80,82]$. The available data demonstrated that CHM plus Glu significantly reduced the Lequesne index when compared to Glu alone $(\mathrm{SMD}=-0.734 ; 95 \% \mathrm{CI}=-0.890$ to $-0.579 ; \quad P \leq 0.001$; heterogeneity $\chi^{2}=11.74, \mathrm{df}=5, I^{2}=$ $57.4 \%, P=0.038$, Figure $7(\mathrm{c}))$. Based on the Lequesne index, four studies compared CHM versus Glu [35, 62, 69, 76]. The pooled results indicated that CHM monotherapy significantly reduced the Lequesne index when compared to Glu alone $(\mathrm{SMD}=-1.071 ; 95 \% \mathrm{CI}=-1.283$ to $-0.859 ; P \leq 0.001$; het erogeneity $\chi^{2}=3.61, \quad \mathrm{df}=3, \quad I^{2}=17.0 \%, \quad P=0.306$, Figure $7(\mathrm{~d}))$.

3.3.5. Effective Rate. Eighteen studies examined the effectiveness of CHM plus NSAIDS to NSAIDS alone [34, 37, 38, 42, 44, 46, $53,55,57,60,65,67,68,75,79,81,87]$. The pooled data demonstrated that CHM plus NSAIDS significantly increased the effective rate when compared to NSAIDS alone $(\mathrm{RR}=1.247$; $95 \% \mathrm{CI}=1.192$ to $1.303 ; P \leq 0.001$; heterogeneity $\chi^{2}=16.86$, $\mathrm{df}=17, I^{2}=0 \%, P=0.464$, Figure $\left.8(\mathrm{a})\right)$. There were ten studies comparing the effective rate between CHM and NSAIDS [50, $52,54,56,61,71,73,77,83,85]$. The pooled data indicated that CHM monotherapy significantly improved the effective rate when compared to NSAIDS alone $(\mathrm{RR}=1.154 ; 95 \% \mathrm{CI}=$ 1.084 to $1.229 ; P \leq 0.001$; heterogeneity $\chi^{2}=9.20, \mathrm{df}=9, I^{2}=$ $2.2 \%, P=0.419$, Figure $8(\mathrm{~b}))$. Ten studies compared the effective rate between CHM plus Glu with Glu [33, 40, 45, 47-49, $59,72,80,82]$. The pooled data demonstrated that CHM plus Glu significantly increased the effective rate when compared to Glu alone $(\mathrm{RR}=1.223 ; 95 \% \mathrm{CI}=1.156$ to $1.295 ; P \leq 0.001$; heterogeneity $\chi^{2}=4.21, \quad \mathrm{df}=9, \quad I^{2}=0 \%, \quad P=0.897$, Figure $8(\mathrm{c})$ ). Ten studies compared the effective rate of CHM and Glu treatment $[35,43,51,62,63,69,70,76,84,86]$. The pooled data demonstrated that CHM monotherapy significantly increased the effective rate when compared to Glu alone $(\mathrm{RR}=1.208 ; 95 \% \mathrm{CI}=1.148$ to $1.272 ; P \leq 0.001$; heterogeneity $\chi^{2}=12.03, \mathrm{df}=9, I^{2}=25.2 \%, P=0.212$, Figure $\left.8(\mathrm{~d})\right)$. 


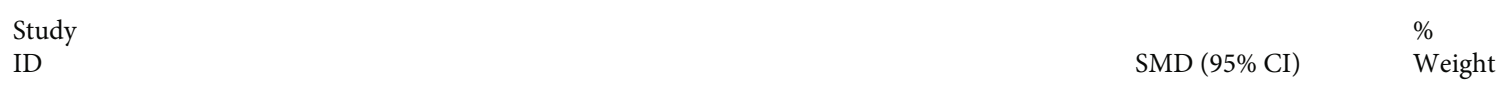

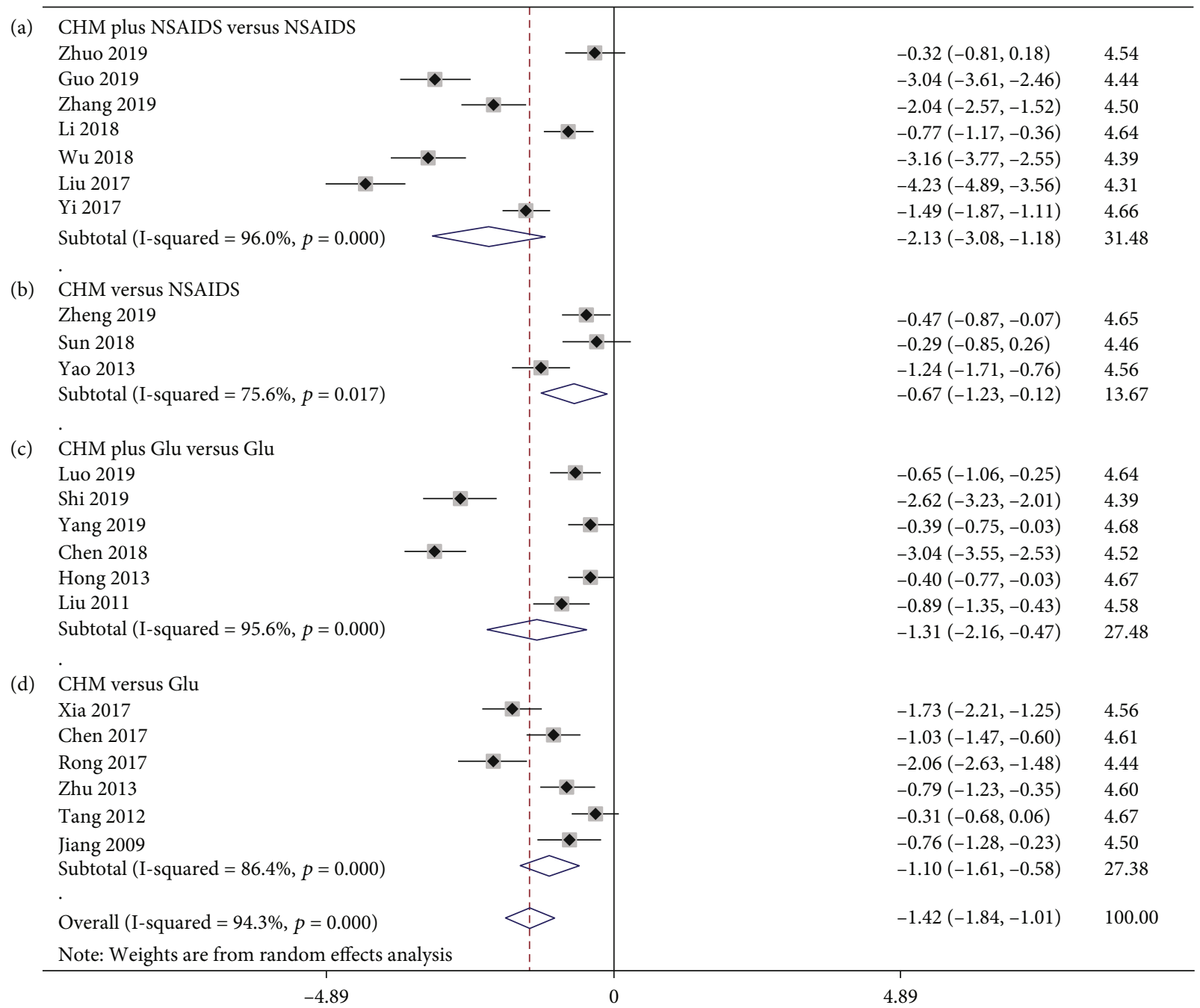

FIGUre 4: Forest plot of WOMAC: (a) CHM plus NSAIDS versus NSAIDS, (b) CHM versus NSAIDS, (c) CHM plus Glu versus Glu, and (d) $\mathrm{CHM}$ versus Glu.

3.3.6. Adverse Events. We identified adverse events in thirtytwo studies. The trial group experienced 108/1489 adverse events, whereas the control group experienced 172/1472. The risk of adverse events was significantly lower in the $\mathrm{CHM}$ group than that in the control group $(\mathrm{RR}=0.625 ; 95$ $\% \mathrm{CI}=0.500$ to $0.783 ; P \leq 0.001 ;$ heterogeneity $\chi^{2}=40.94$, $\mathrm{df}=31, I^{2}=24.3 \%, P=0.109$, Figure 9). Our findings indicated that the most often occurring adverse effects included gastrointestinal discomfort (nausea, diarrhea, thirst, poor appetite, stomach pain, and constipation), abnormal liver function, and rash. Significant adverse impacts that were mild, no severe adverse impacts, and death were reported in the included RCTs.

3.3.7. Publication Bias and Sensitivity Analysis. We examined the possibility of publication bias of the adverse effects in this meta-analysis using Begg's funnel plot and Egger's test (Figure 10). As a result of the symmetrical shape of the funnel plots and the $P$ values from Begg's and Egger's tests, there was evidence of notable publication bias for adverse events $(P=0.661$ and $P=0.847$, respectively).

To establish the influence of each included study on the pooled RRs for the effective rate and adverse effects and to validate the robustness of our findings, we performed a sensitivity analysis by excluding one study at a time and computing the pooled RRs for the rest of the RCTs. The results of the sensitivity analysis indicated that excluding each study individually had no discernible influence on the pooled RRs showing that the findings of this meta-analysis are comparatively robust (Figure 11).

3.3.8. Description of the CHMs. The most commonly used herbs across all formulae included Niu Xi (Radix Achyranthis Bidentatae, Twotooth Achyranthes root), Di Huang (Radix Rehmanniae, Rehmannia root), Dang Gui (Radix Angelicae Sinensis, Chinese angelica), Chuan Xiong (Radix Ligustici Wallichii, Sichuan lovage rhizome), Du Zhong (Cortex Eucommiae Ulmoidis, Epimedium), Gan Cao (Radix Glycyrrhizae, 


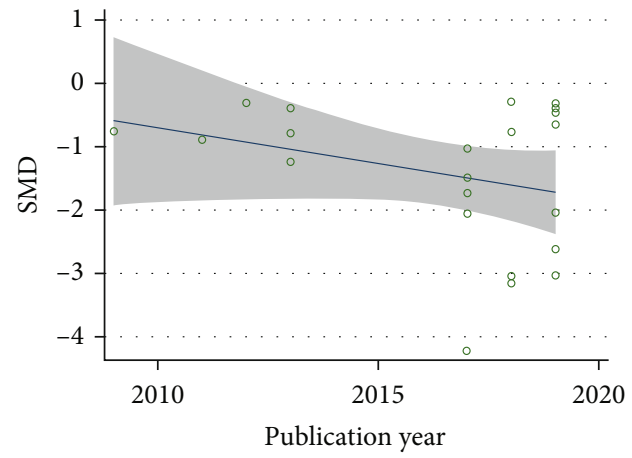

(a)

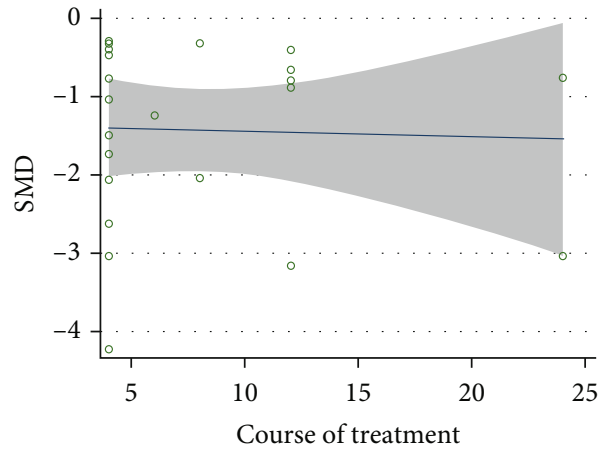

(b)

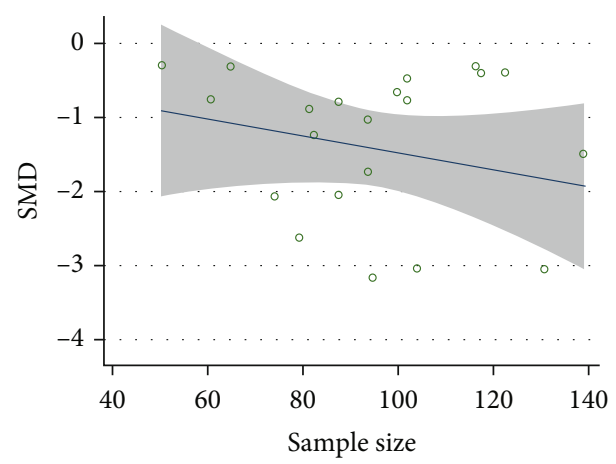

(c)

FIGURE 5: Metaregression analysis of WOMAC for (a) publication year, (b) course of treatment, (c) and sample size.

Study

ID

(a) CHM plus NSAIDS versus NSAIDS

Mo 2018

Li 2018

Liu 2017

Yi 2017

Ren 2016

Subtotal (I-squared $=96.0 \%, p=0.000)$

(b) CHM versus NASIDS

Ren 2018

Yuan 2017

Zhou 2012

Subtotal (I-squared $=78.9 \%, p=0.009)$

Overall (I-squared $=94.9 \%, p=0.000)$

Note: weights are from random effects analysis

$-5.16$
$\operatorname{SMD}(95 \% \mathrm{CI})$

$\%$

Weight

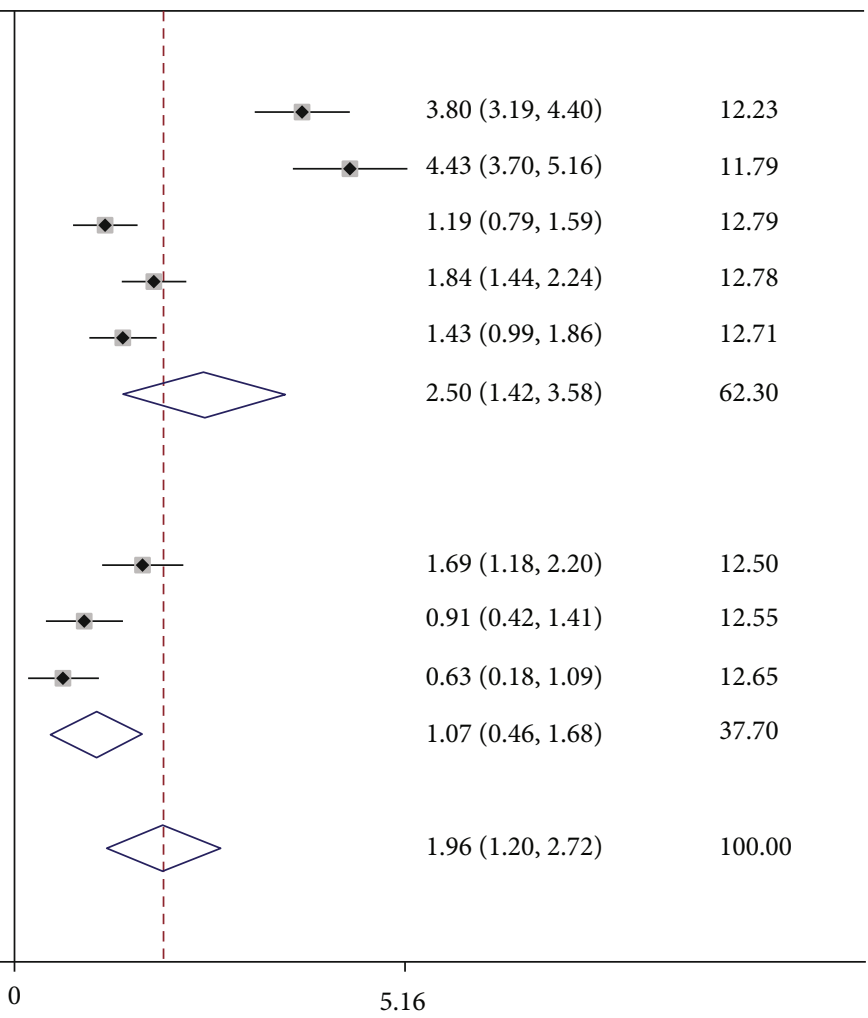

FIGURE 6: Forest plot of Lysholm score: (a) CHM plus NSAIDS versus NSAIDS and (b) CHM versus NSAIDS. 
Study

ID
$\operatorname{SMD}(95 \% \mathrm{CI}) \quad$ Weight

$-0.75(-1.21,-0.29) \quad 5.19$

$-0.78(-1.28,-0.27) \quad 4.24$

$-1.26(-1.80,-0.72) \quad 3.73$

$-1.03(-1.46,-0.60) \quad 5.96$

$-0.67(-1.12,-0.22) \quad 5.40$

$-0.88(-1.09,-0.67) \quad 24.51$

$-0.80(-1.24,-0.37) \quad 5.80$

$-0.80(-1.24,-0.37) \quad 5.80$

$-0.75(-1.13,-0.36) \quad 7.33$

$-1.08(-1.45,-0.71) \quad 7.96$

$-0.30(-0.61,0.02) \quad 11.00$

$-0.74(-1.19,-0.29) \quad 5.34$

$-0.90(-1.27,-0.52) \quad 7.77$

$-0.85(-1.29,-0.42) \quad 5.88$

$-0.73(-0.89,-0.58) \quad 45.27$

$-1.42(-1.85,-0.98) \quad 5.73$

$-0.88(-1.41,-0.35) \quad 3.89$

$-1.07(-1.45,-0.68) \quad 7.48$

$-0.90(-1.29,-0.52) \quad 7.30$

$-1.07(-1.28,-0.86) \quad 24.41$

Heterogeneity between groups: $p=0.094$

Overall (I-squared $=40.9 \%, p=0.045)$

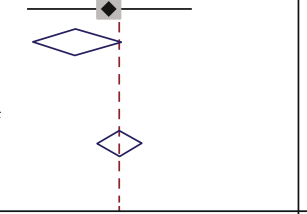

$-0.86(-0.96,-0.75) \quad 100.00$

$-1.85$

1.85

Figure 7: Forest plot of Lequesne index: (a) CHM plus NSAIDS versus NSAIDS, (b) CHM versus NSAIDS, (c) CHM plus Glu versus Glu, and (d) CHM versus Glu.

liquorice), Du Huo (Radix Angelicae Pubescentis, Pubescent angelica root), Sang Ji Sheng (Radix Loranthi Seu visci, Chinese taxillus twig), Bai Shao (Radix Paeoniae Alba, White paeony root), Fang Feng (Radix Ledebouriellae Divaricatae, Divaricate saposhnikovia root), Qin Jiao (Radix Gentianae Macrophyllae, Largeleaf gentian root), Fu Ling (Poria Cocos Wolff, Tuckahoe), Xi Xin (Asari Radix et Rhizoma, Asarum), Yin Yang Huo (Epimedium brevicornu Maxim, Icariin), Huang Qi (Radix Astragali Membranacei, Astragalus), Wei Ling Xian (Radix Clematidis Chinensis, Chinese clematis root), and $\mathrm{Bu} \mathrm{Gu}$ Zhi (Psoralea corylifolia Linn, Fructus psoraleae) (Table 4).

\section{Discussion}

4.1. Summary of Evidence. Herein, we updated a systematic review and meta-analysis on the efficacy and safety of CHM treatment in patients with KOA. A total of fifty-six highquality RCTs, including 5350 patients with $\mathrm{KOA}$, were included in the analysis. Our primary findings indicated that using $\mathrm{CHM}$ as adjuvant therapy or monotherapy for $\mathrm{KOA}$ treatment reduced the VAS, WOMAC, and Lequesne index while improving the Lysholm score and overall effective rate. Additionally, we discovered that CHM adjuvant or monotherapy had fewer adverse effects than the controls, indicating that
CHM was safe and effective in treating KOA. Therefore, we provide supporting evidence that, to a significant extent, $\mathrm{CHM}$ can potentially be recommended for use in $\mathrm{KOA}$ patients.

4.2. Comparison with Previous Studies. Significant research demonstrates that the oral and topical use of CHM is both safe and effective in the treatment of KOA. A meta-analysis of 23 RCTs including 2362 patients demonstrated that $\mathrm{CHM}$ is both safe and effective in alleviating pain, restoring function, and promoting health in patients with KOA [28]. Another systematic review found that Duhuo Jisheng decoction (DJD) combined with Western medicine or sodium hyaluronate injection was effective in treating KOA [88]. However, the effectiveness and safety of DJD remain debatable due to a scarcity of clinical trials and a lack of methodological rigor. Additionally, a Cochrane review of two RCTs including 327 patients found that orally bioavailable avocado soybean unsaponifiables (ASU) significantly relieved the pain symptoms in hip-OA patients when compared to a placebo. Additionally, this review established that the use of ASU helped patients in reducing their use of NSAIDs [89]. However, a primary concern in these earlier investigations has been the limited sample size and low quality. Therefore, in the current systematic review, we included 56 
Study

$\%$ ID

RR (95\% CI)
Weight (a) CHM plus NSAIDS versus NSAIDS Qian 2019

Wang 2019

Zhuo 2019

Guo 2019

Zhang 2019

Wu 2018

Mo 2018

Li 2018

Wu 2018

Fu 2018

Cui 2018

Liu 2017

Yi 2017

Zhang 2017

Song 2017

Ren 2016

Yi 2017

Huang 2015

Subtotal $(\mathrm{I}$-squared $=0.0 \%, p=0.464)$

(b) CHM versus NSAIDS

Zheng 2019

Ma 2018

Ren 2018

Yuan 2017

Sun 2018

Pan 2017

Yang 2016

Yao 2013

Zhou 2012

Ma 2009

Subtotal $(\mathrm{I}$-squared $=2.2 \%, p=0.419)$

(c) CHM plus Glu versus Glu

Luo 2019

Shi 2019

Yang 2019

Zhang 2018

Chen 2018

Lu 2016

Liu 2016

Zheng 2014

Hong 2013

Liu 2011

Subtotal (I-squared $=0.0 \%, p=0.897)$

(d) $\mathrm{CHM}$ versus Glu

Xia 2017

Ma 2019

Zhong 2017

Cui 2017

Tan 2014

Zhu 2013

Wu 2012

Tang 2012

Yu 2010

Jiang 2009

Subtotal (I-squared $=25.2 \%, p=0.212$ )

Overall (I-squared $=1.3 \%, p=0.448)$
$1.31(1.02,1.68)$

$1.18(0.94,1.47)$

$1.25(1.00,1.56)$

$1.28(1.05,1.56)$

$1.21(1.02,1.43)$

$1.25(1.02,1.53)$

$1.18(1.05,1.33)$

$1.81(1.37,2.38)$

$1.30(1.07,1.59)$

$1.12(0.98,1.28)$

$1.10(0.96,1.26)$

$1.21(1.02,1.43)$

$1.22(1.04,1.42)$

$1.25(1.02,1.53)$

$1.26(0.99,1.60)$

$1.30(1.07,1.57)$

$1.15(0.97,1.36)$

$1.42(1.12,1.82)$

$1.25(1.19,1.30)$

$1.03(0.84,1.26)$

$1.29(1.03,1.61)$

$1.28(1.03,1.57)$

$1.19(0.96,1.46)$

$1.05(0.84,1.31)$

$1.03(0.90,1.18)$

$1.16(0.97,1.39)$

$1.16(0.97,1.38)$

$1.08(0.94,1.23)$

$1.34(1.05,1.72)$

$1.15(1.08,1.23)$

$1.26(1.08,1.54)$

$1.23(1.02,1.49)$

$1.17(1.01,1.35)$

$1.22(1.02,1.45)$

$1.23(1.05,1.44)$

$1.22(1.01,1.47)$

$1.27(0.99,1.61)$

$1.31(1.07,1.61)$

$1.28(1.07,1.53)$

$1.09(0.95,1.25)$

$1.22(1.16,1.30)$

1.52

1.91

1.40

2.10

1.99

1.38

2.92

1.52

1.95

1.93

2.98

2.51

2.98

1.43

1.85

2.01

2.69

1.52

36.59

2.28

1.31

1.70

1.58

1.23

2.10

2.16

1.89

2.00

2.05

18.29

2.05

1.75

2.81

2.40

2.81

2.16

1.75

1.70

2.43

2.05

21.90

$1.26(1.05,1.53) \quad 1.99$

$1.10(1.00,1.20) \quad 4.21$

$1.22(1.05,1.42) \quad 2.63$

$1.25(1.04,1.51) \quad 2.18$

$1.45(1.12,1.88) \quad 1.17$

$1.17(1.00,1.37) \quad 2.05$

$1.09(0.99,1.21) \quad 3.10$

$1.36(1.07,1.73) \quad 2.01$

$1.17(1.03,1.33) \quad 2.72$

$1.35(1.02,1.79) \quad 1.17$

$1.21(1.15,1.27) \quad 23.22$

$1.22(1.18,1.25) \quad 100.00$

.42

FIGURE 8: Forest plot of effective rate: (a) CHM plus NSAIDS versus NSAIDS, (b) CHM versus NSAIDS, (c) CHM plus Glu versus Glu, and (d) CHM versus Glu. 


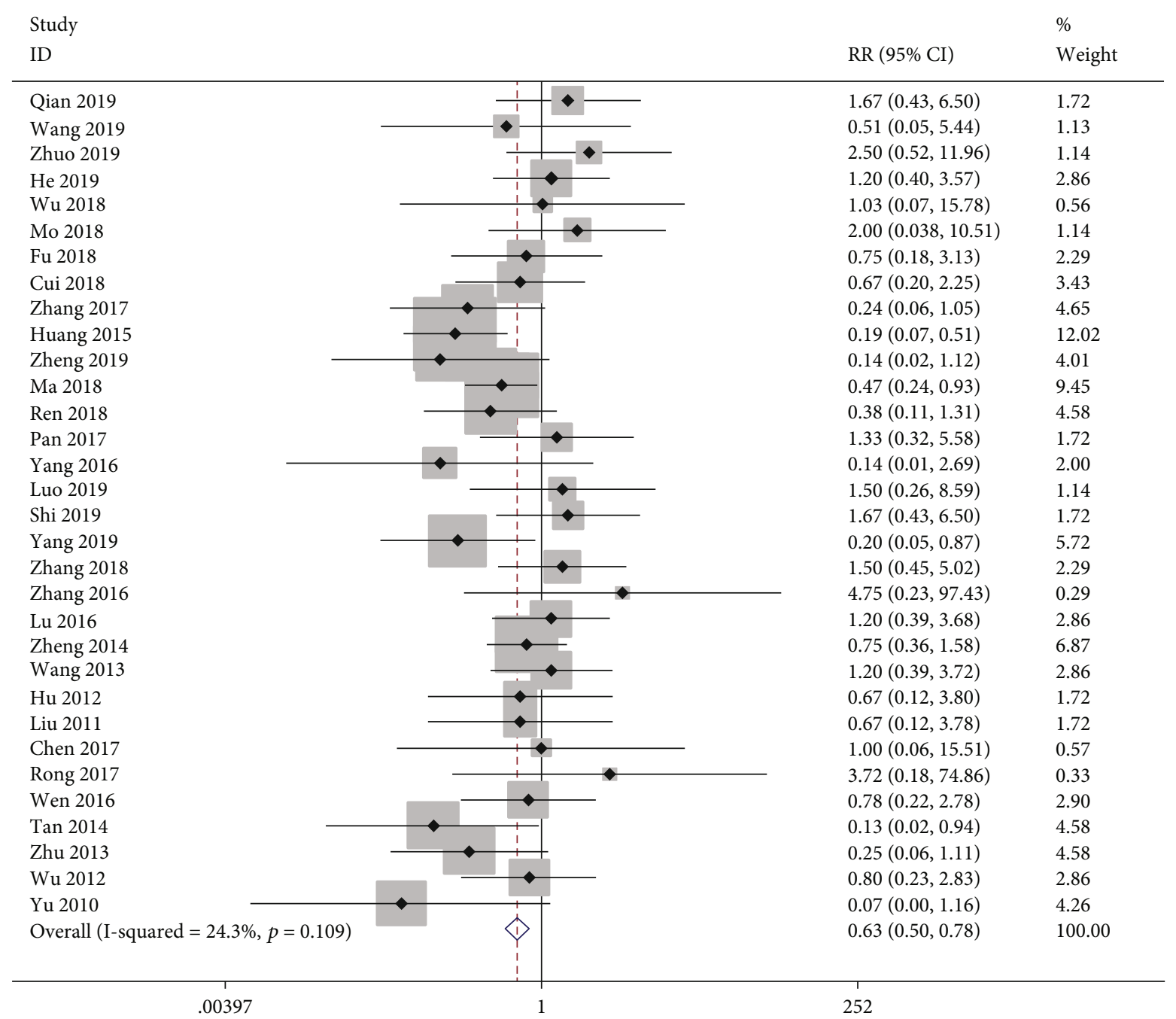

FIgURE 9: Forest plot of adverse events.

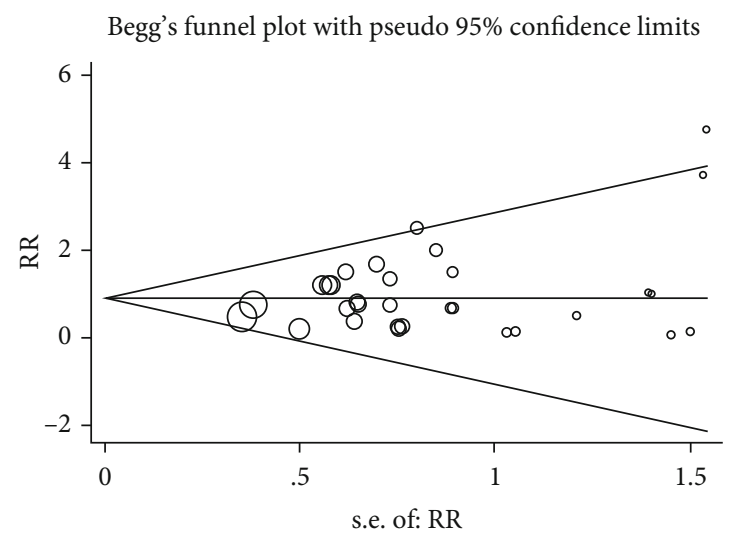

(a)

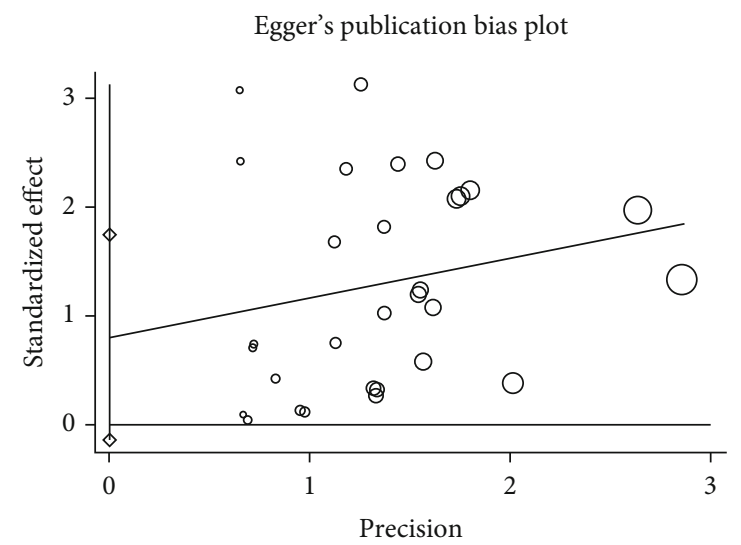

(b)

Figure 10: Begg's funnel plot (a) and Egger's test (b) of adverse events. 


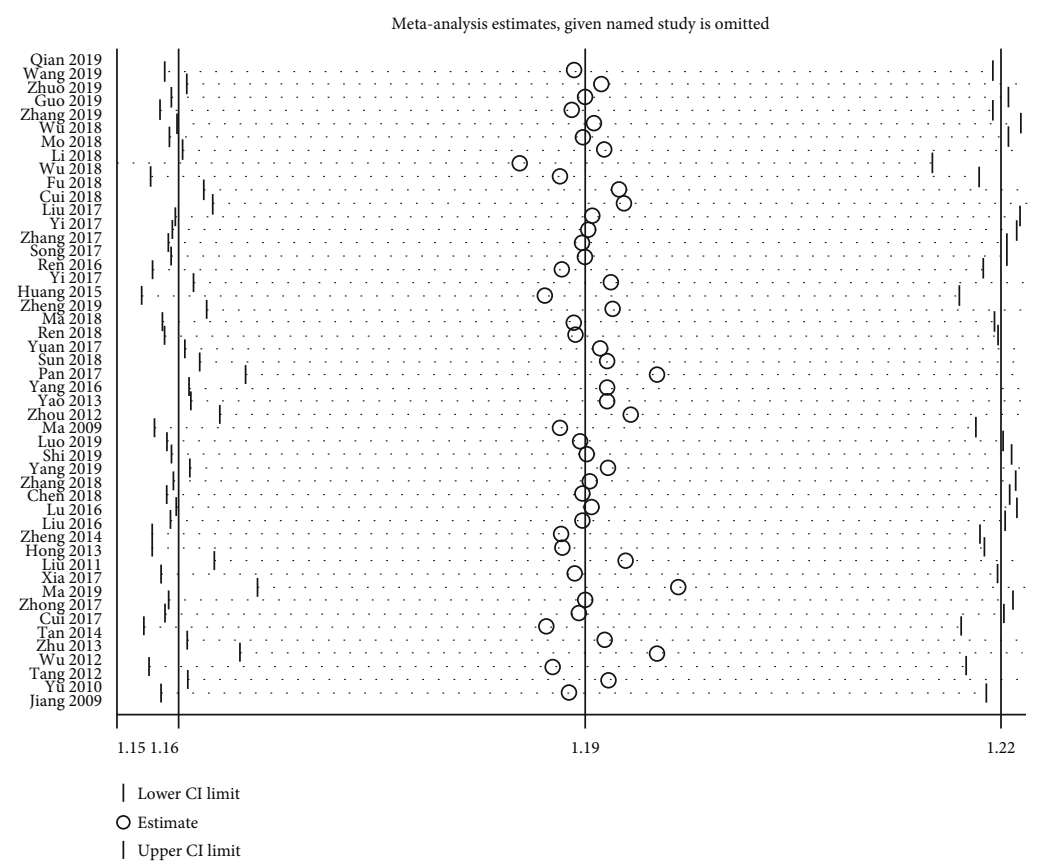

(a)

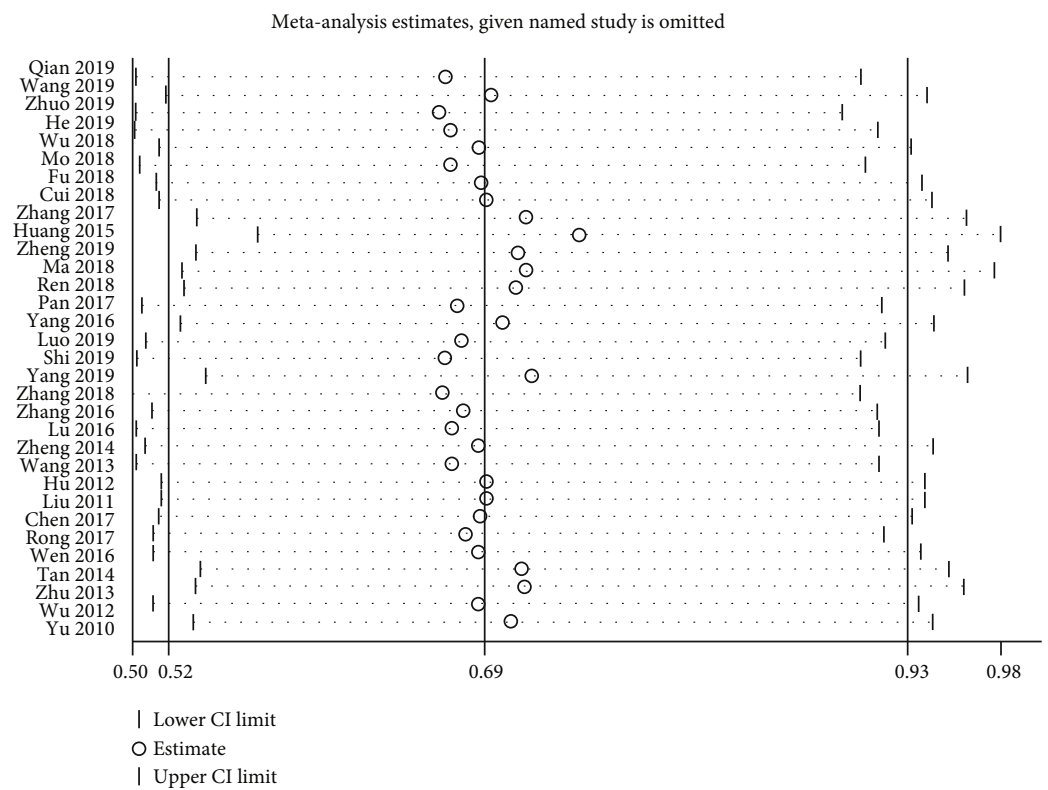

(b)

FIGURE 11: Sensitivity analysis for effective rate (a) and adverse events (b).

high-quality RCTs involving 5350 patients with KOA, which provides strong evidence that CHM is safe and effective for patients with KOA, consistent with the previous studies.

4.3. Strengths. The strengths of this meta-analysis study included a clearly defined research question, which minimized the bias in the selection of RCTs and improved the fidelity and consistency due to a precise research approach that we designed before the meta-analysis, an in-depth search of the literature, agreement between the two researchers on the entry data components, and quality control appraisal of all data. All of the studies included were RCTs with a significant propor- tion being of high quality. This assisted in overcoming the drawbacks associated with recall or selection bias in nonrandomized studies. Additionally, the total number of trials and the overall sample size were comparatively large ( 56 trials with 5350 patients). To ascertain the source of heterogeneity, we performed subgroup and metaregression analyses. Consequently, we found no evidence of publication bias in this meta-analysis, and sensitivity analysis revealed that the findings of this meta-analysis are comparatively robust.

4.4. Limitations. This study has several limitations. First, although RCTs were included, the primary studies included 
TABLE 4: Frequently used herbs in included studies.

\begin{tabular}{lcccc}
\hline Chinese name & Latin name & English name & Family & Number of studies (\%) \\
\hline Niu xi & Radix Achyranthis Bidentatae & Twotooth Achyranthes root & Amaranthaceae & $33(58.93 \%)$ \\
Di Huang & Radix Rehmanniae & Rehmannia root & Scrophulariaceae & $30(53.57 \%)$ \\
Dang Gui & Radix Angelicae Sinensis & Chinese angelica & Apiaceae & $29(51.79 \%)$ \\
Chuan Xiong & Radix Ligustici Wallichii & Sichuan lovage rhizome & Apiaceae & $29(51.79 \%)$ \\
Du Zhong & Cortex Eucommiae Ulmoidis & Epimedium & Eucommiaceae & $26(46.43 \%)$ \\
Gan Cao & Radix Glycyrrhizae & Liquorice & Papilionaceae & $25(44.64 \%)$ \\
Du Huo & Radix Angelicae Pubescentis & Pubescent angelica root & Apiaceae & $21(37.50 \%)$ \\
Sang Ji sheng & Radix Loranthi Seu visci & Chinese taxillus twig & Loranthaceae & $21(37.50 \%)$ \\
Bai shao & Radix Paeoniae Alba & White paeony root & Asclepiadaceae & $19(33.93 \%)$ \\
Fang Feng & Radix Ledebouriellae Divaricatae & Divaricate saposhnikovia root & Apiaceae & $18(32.14 \%)$ \\
Qin Jiao & Radix Gentianae Macrophyllae & Largeleaf gentian root & Gentianaceae & $18(32.14 \%)$ \\
Fu Ling & Poria Cocos Wolff & Tuckahoe & Polyporaceae & $17(30.36 \%)$ \\
Xi Xin & Asari Radix et Rhizoma & Asarum & Aristolochiaceae & $16(28.57 \%)$ \\
Yin Yang Huo & Epimedium brevicornu Maxim & Icariin & Berberidaceae & $11(19.64 \%)$ \\
Huang qi & Radix Astragali Membranacei & Astragalus & Leguminosae & $11(19.64 \%)$ \\
Wei Ling Xian & Radix Clematidis Chinensis & Chinese clematis root & Ranunculaceae & $11(19.64 \%)$ \\
Bu Gu Zhi & Psoralea corylifolia Linn & Fructus psoraleae & Leguminosae & $11(19.64 \%)$ \\
\hline
\end{tabular}

had certain inherent and methodological limitations; specifically, only 42 of the trials supplied sufficient information on the randomization process. The remaining RCTs, on the other hand, reported the allocation concealment. KOA is a chronic condition requiring lifelong treatment. Long-term efficacy and safety studies are critical for determining a drug's therapeutic usefulness. However, the duration of therapy, in this case, was between two and twelve weeks. Therefore, we were unable to assess the long-term safety of CHM for treating KOA since the duration of treatment in the included studies was short, and no dropouts were revealed in a significant percentage of the included studies. Thirdly, the formula composition, dosage, administration approaches, and duration of CHM treatments varied significantly in the primary RCTs. This clinical heterogeneity has the potential to jeopardize the validity of our findings. Fourthly, a significant proportion of the included RCTs did not involve a formal pretrial sample size calculation. Inadequate sample size in RCTs appears to be one risk factor for overestimating intervention benefits. Finally, we limited our search to studies published in English or Chinese repositories; therefore, studies published in other languages may have been overlooked. Additionally, because all RCTs included in the study were conducted in China, our findings may not be generalizable. Therefore, more multicenter RCTs of CHM for treating KOA are required to allow for global data generalization.

4.5. Implications for Practice. The evidence presented here indicates that using CHM as monotherapy or adjuvant treatment is beneficial and typically safe for treating KOA patients. We identified Radix Achyranthis Bidentatae, Radix Rehmanniae, Radix Angelicae Sinensis, Radix Ligustici Wallichii, Cortex Eucommiae Ulmoidis, Radix Glycyrrhizae, Radix Angelicae Pubescentis, Radix Loranthi Seu visci, Radix Paeoniae Alba, Radix Ledebouriellae Divaricatae, Radix Gentianae
Macrophyllae, Poria Cocos Wolf, Asari Radix et Rhizoma, Epimedium brevicornu Maxim, Radix Astragali Membranacei, Radix Clematidis Chinensis, and Psoralea corylifolia Linn as the most frequently used herbs in KOA prescriptions., which should further be considered in the formulation of Chinese herbal prescriptions for KOA. Therefore, based on the high frequency of use of $\mathrm{CHM}$ for $\mathrm{KOA}$, their therapeutic principles can guide CHM treatment for KOA, thereby increasing its effectiveness and safety.

4.6. Implications for Research. Here, we provide key concepts that are likely to stimulate further research in this field. Initiatives to increase the methodological quality of RCTs are urgently needed. We urge that in the future, recommendations such as the CONSORT Extension for Chinese Herbal Medicine Formulas 2017 [90], the CONSORT 2010 statement [91], and the protocols for designing RCTs to investigate CHM [92] be used to establish and report RCTs on CHM. Despite the finding that CHM therapy was reasonably safe for patients with KOA in the evaluated studies, further research is needed to corroborate the safety of CHM for KOA. Bian et al. [93] established a standard format for reporting adverse drug reactions (ADR) in CHM, which is likely to enhance ADR reporting. Clinical trials and studies with a longer follow-up time are recommended to provide a complete understanding of the long-term safety profile of $\mathrm{CHM}$ in patients with KOA. Recent advances in integrative medicine have enabled research to be conducted on disease-syndrome combinations. The effectiveness of TCM practice is contingent upon accurate syndrome differentiation. Therefore, an excellent distinction of disease symptoms is required for drug prescriptions [94]. Accurate syndrome differentiation of KOA should be performed during the evaluation of the safety and efficacy of CHM treatment. Individualized TCM prescriptions will give satisfactory treatment 
for specific diseases. For example, a study by Bensoussan et al. [95] published in JAMA showed that using personalized CHM to treat irritable bowel syndrome was superior to common hypnotic prescriptions. Thus, in future clinic practice, a suitable selection of medications among the 17 most often used herbs is recommended based on syndrome-specific characteristics. This will improve the efficacy of CHM in the treatment of KOA.

\section{Conclusion}

Our systematic and meta-analysis study offers supportive evidence that $\mathrm{CHM}$, either adjuvant therapy or monotherapy, reduces the VAS, WOMAC, and Lequesne index and improves the Lysholm score and overall effective rate in patients with KOA. Additionally, CHM was well tolerated and safe in KOA patients. We found frequently used CHMs that might contribute to the formulation of a herbal formula that could be considered for further clinical use. However, given the heterogeneity and limited sample size in this study, larger multicenter and high-quality RCTs are needed to validate the benefits of CHM in the treatment of KOA.

$\begin{array}{ll}\text { Abbreviations } \\ \text { ADR: } & \text { Adverse drug reactions } \\ \text { RCT: } & \text { Randomized controlled trial } \\ \text { TG: } & \text { Trial group } \\ \text { CG: } & \text { Control group } \\ \text { COA: } & \text { Chinese Orthopedic Association } \\ \text { CRA: } & \text { Chinese Rheumatology Association } \\ \text { ACR: } & \text { American College of Rheumatology } \\ \text { VAS: } & \text { Visual analog score } \\ \text { WOMAC: } & \text { Western Ontario and McMaster Universities } \\ & \text { Osteoarthritis Index } \\ \text { ER: } & \text { Effective rate } \\ \text { NR: } & \text { Not reported } \\ \text { CHM: } & \text { Chinese herbal medicine } \\ \text { KOA: } & \text { Knee osteoarthritis } \\ \text { NSAIDS: } & \text { Nonsteroidal anti-inflammatory drugs } \\ \text { PRISMA: } & \text { Preferred Reporting Items for Systematic } \\ & \text { Reviews and Meta-Analyses } \\ \text { WCM: } & \text { Western conventional medication } \\ \text { TCM: } & \text { Traditional Chinese medicine } \\ \text { SMD: } & \text { Standard mean difference } \\ \text { RR: } & \text { Relative risk } \\ \text { DJD: } & \text { Duhuo Jisheng decoction } \\ \text { ASU: } & \text { Avocado soybean unsaponifiables. } \\ & \end{array}$

\section{Data Availability}

Previously reported data were used to support this study. These prior studies and datasets are cited at relevant places within the text as references [28-83].

\section{Conflicts of Interest}

The authors declare that there is no conflict of interests regarding the publication of this study.

\section{Authors' Contributions}

J.J.L. is responsible for conceptualization, methodology, and software. J.J.Z. is responsible for data curation and writing of the initial draft. J.R.C. is responsible for visualization and investigation. M.M.C. is responsible for supervision.: Z.L. is responsible for software and validation. Z.L. is responsible for writing-reviewing and editing.

\section{Acknowledgments}

The authors thank Home for Researchers (http://www.homefor-researchers.com) for their help with language editing.

\section{Supplementary Materials}

PubMed search strategies and graphical abstract were provided as supplementary material. (Supplementary Materials)

\section{References}

[1] G. R. Squires, S. Okouneff, M. Ionescu, and A. R. Poole, “The pathobiology of focal lesion development in aging human articular cartilage and molecular matrix changes characteristic of osteoarthritis," Arthritis and Rheumatism, vol. 48, no. 5, pp. 1261-1270, 2003.

[2] E. Halilaj, D. C. Moore, D. H. Laidlaw et al., "The morphology of the thumb carpometacarpal joint does not differ between men and women, but changes with aging and early osteoarthritis," Journal of Biomechanics, vol. 47, no. 11, pp. 27092714, 2014.

[3] D. J. Hunter and D. T. Felson, "Osteoarthritis," BMJ, vol. 332, no. 7542, pp. 639-642, 2006.

[4] R. C. Lawrence, D. T. Felson, C. G. Helmick et al., "Estimates of the prevalence of arthritis and other rheumatic conditions in the United States. Part II," Arthritis and Rheumatism, vol. 58, no. 1, pp. 26-35, 2008.

[5] V. Silverwood, M. Blagojevic-Bucknall, C. Jinks, J. L. Jordan, J. Protheroe, and K. P. Jordan, "Current evidence on risk factors for knee osteoarthritis in older adults: a systematic review and meta-analysis," Osteoarthritis and Cartilage, vol. 23, no. 4, pp. 507-515, 2015.

[6] D. J. Barten, L. C. Swinkels, S. A. Dorsman, J. Dekker, C. Veenhof, and D. H. de Bakker, "Treatment of hip/knee osteoarthritis in Dutch general practice and physical therapy practice: an observational study," BMC Family Practice, vol. 16, no. 1, 2015.

[7] A. D. Woolf and B. Pfleger, "Burden of major musculoskeletal conditions," Bulletin of the World Health Organization, vol. 81, no. 9, pp. 646-656, 2003.

[8] B. S. Serni, G. Verbruggen, I. Zimmerman-Gorska et al., "EULAR Recommendations 2003: an evidence based approach to the management of knee osteoarthritis: report of a Task Force of the Standing Committee for International Clinical Studies Including Therapeutic Trials (ESCISIT)," Annals of the Rheumatic Diseases, vol. 62, no. 12, pp. 1145-1155, 2003.

[9] Z. Jotanovic, R. Mihelic, B. Sestan, and Z. Dembic, "Emerging pathways and promising agents with possible disease modifying effect in osteoarthritis treatment," Current Drug Targets, vol. 15, no. 6, pp. 635-661, 2014. 
[10] M. C. Hochberg, R. D. Altman, K. T. April et al., "American College of Rheumatology 2012 recommendations for the use of nonpharmacologic and pharmacologic therapies in osteoarthritis of the hand, hip, and knee," Arthritis Care \& Research, vol. 64, no. 4, pp. 465-474, 2012.

[11] L. Fernandes, K. B. Hagen, J. W. Bijlsma et al., "EULAR recommendations for the non-pharmacological core management of hip and knee osteoarthritis," Annals of the Rheumatic Diseases, vol. 72, no. 7, pp. 1125-1135, 2013.

[12] G. A. Brown, "AAOS clinical practice guideline: treatment of osteoarthritis of the knee: evidence-based guideline, 2nd edition," The Journal of the American Academy of Orthopaedic Surgeons, vol. 21, no. 9, pp. 577-579, 2013.

[13] J. N. Katz, K. R. Arant, and R. F. Loeser, "Diagnosis and treatment of hip and knee Osteoarthritis," JAMA, vol. 325, no. 6, pp. 568-578, 2021.

[14] M. S. M. Persson, J. Stocks, G. Varadi et al., "Predicting response to topical non-steroidal anti-inflammatory drugs in osteoarthritis: an individual patient data meta-analysis of randomized controlled trials," Rheumatology, vol. 59, no. 9, pp. 2207-2216, 2020.

[15] D. G. Wolff, C. Christophersen, S. M. Brown, and M. K. Mulcahey, "Topical nonsteroidal anti-inflammatory drugs in the treatment of knee osteoarthritis: a systematic review and meta-analysis," The Physician and Sportsmedicine, vol. 49, no. 4, pp. 381-391, 2021.

[16] M. N. Essex, M. A. O'Connell, R. Behar, and W. Bao, "Efficacy and safety of nonsteroidal anti-inflammatory drugs in Asian patients with knee osteoarthritis: summary of a randomized, placebo-controlled study," International Journal of Rheumatic Diseases, vol. 19, no. 3, pp. 262-270, 2016.

[17] A. Askari, S. A. Ravansalar, M. M. Naghizadeh et al., "The efficacy of topical sesame oil in patients with knee osteoarthritis: a randomized double-blinded active-controlled non-inferiority clinical trial," Complementary Therapies in Medicine, vol. 47, p. 102183, 2019.

[18] M. Anvari, H. Dortaj, B. Hashemibeni, and M. Pourentezari, "Application of some herbal medicine used for the treatment of osteoarthritis and chondrogenesis," Traditional and Integrative Medicine, vol. 5, 2020.

[19] Z. Chen, X. Ye, Y. Wang et al., "The efficacy of backward walking on static stability, proprioception, pain, and physical function of patients with knee osteoarthritis: a randomized controlled trial," Evidence-Based Complementary and Alternative Medicine, vol. 2021, Article ID 5574966, 9 pages, 2021.

[20] L. Lao, M. Hochberg, D. Y. W. Lee et al., "Huo-Luo-Xiao-Ling (HLXL)-Dan, a traditional Chinese medicine, for patients with osteoarthritis of the knee: a multi-site, randomized, doubleblind, placebo-controlled phase II clinical trial," Osteoarthritis and Cartilage, vol. 23, no. 12, pp. 2102-2108, 2015.

[21] R. Chen, M. Chen, T. Su et al., "Heat-sensitive moxibustion in patients with osteoarthritis of the knee: a three-armed multicentre randomised active control trial," Acupuncture in Medicine, vol. 33, no. 4, pp. 262-269, 2015.

[22] J. W. Bost, A. Maroon, and A. Maroon, "Natural antiinflammatory agents for pain relief," Surgical Neurology International, vol. 1, no. 1, 2010.

[23] Y. Zhang, Z. Z. Wang, H. M. Sun, P. Li, Y. F. Li, and N. H. Chen, "Systematic review of traditional Chinese medicine for depression in Parkinson's disease," The American Journal of Chinese Medicine, vol. 42, no. 5, pp. 1035-1051, 2014.
[24] W. Ling, Y. Huang, J. H. Xu et al., "Consistent efficacy of Wendan decoction for the treatment of digestive reflux disorders," The American Journal of Chinese Medicine, vol. 43, no. 5, pp. 893-913, 2015.

[25] D. Zhou, B. Liu, X. Xiao, P. Dai, S. Ma, and W. Huang, "The effect of safflower yellow on spinal cord ischemia reperfusion injury in rabbits," Oxidative Medicine and Cellular Longevity, vol. 2013, Article ID 692302, 9 pages, 2013.

[26] L. Pan, J. Zhou, H. Zhu et al., "Study on integrated pharmacokinetics of gardenia acid and geniposide: time-antioxidant efficacy after oral administration of Huanglian-Zhizi couplet medicine from Huang-Lian-Jie-Du-Tang in MCAO rats," The American Journal of Chinese Medicine, vol. 42, no. 2, pp. 393-407, 2014.

[27] L. Zhu, S. Yang, S. Wang, H. Gong, L. Li, and X. Wei, "Effectiveness and safety of manufactured Chinese herbal formula for knee osteoarthritis: insights from a systematic review," Evidence-Based Complementary and Alternative Medicine, vol. 2015, Article ID 328642, 19 pages, 2015.

[28] B. Chen, H. Zhan, J. Marszalek et al., "Traditional Chinese medications for knee osteoarthritis pain: a meta-analysis of randomized controlled trials," The American Journal of Chinese Medicine, vol. 44, no. 4, pp. 677-703, 2016.

[29] K. Chan, D. Shaw, M. S. Simmonds et al., "Good practice in reviewing and publishing studies on herbal medicine, with special emphasis on traditional Chinese medicine and Chinese materia medica," Journal of Ethnopharmacology, vol. 140, no. 3, pp. 469-475, 2012.

[30] D. Moher, A. Liberati, J. Tetzlaff, D. G. Altman, and The PRISMA Group, "Preferred reporting items for systematic reviews and meta-analyses: the PRISMA statement," PLoS Medicine, vol. 6, no. 7, 2009.

[31] J. Higgins, "Cochrane Handbook for Systematic Reviews of Interventions Version 5.1.0. The Cochrane Collaboration (Eds)," Naunyn-Schmiedebergs Archiv für experimentelle Pathologie und Pharmakologie, vol. 5, 2011.

[32] S. Chen, "Clinical observation on treatment of knee osteoarthritis with San Bi Xiao granule," Nei Mongol Journal of Traditional Chinese Medicine, vol. 36, no. Z1, pp. 8-10, 2017.

[33] Z. Chen and D. Zhang, "Efficacy of Danqi granule combined with glucosamine sulfate on the treatment of osteoarthritis," Chinese Journal of Osteoporosis, vol. 24, no. 7, pp. 900-903, 2018.

[34] H. Cui, "Clinical observation on Bushen Huoxue recipe combined with diclofenac sodium sustained-release tablets for knee osteoarthritis of kidney deficiency and blood stasis type," Hebei Journal of Traditional Chinese Medicine, vol. 40, no. 2, pp. 246-249, 2018.

[35] S. Cui, "Clinical study on Chaihu Jiangu decoction in the treatment of knee osteoarthritis," Chinese Medicine Modern Distance Education of China, vol. 15, no. 12, pp. 101-103, 2017.

[36] B. Dou, Z. Wang, Y. Xie, and B. Tang, "Summary of 30 cases of knee osteoarthritis treated by Danzikangji granules," Hunan Journal of Traditional Chinese Medicine, vol. 31, no. 5, pp. 78-79, 2015.

[37] P. Fu, Q. Qiu, and J. Li, "Clinical study on the prevention and treatment of early knee osteoarthritis with Duhuo Zhitang decoction and celecoxib capsule," World Journal of Integrated Traditional and Western Medicine, vol. 13, no. 1, pp. 66-69, 2018.

[38] M. Guo, "Effects of modified Duhuo Shengtang decoction on pain and knee function in patients with liver and kidney 
deficiency knee osteoarthritis," Journal of Huaihai Medicine, vol. 37, no. 4, pp. 413-415, 2019.

[39] X. He, X. Lai, and C. Zhong, "Effect of Shuanggusanzi capsule combined with celecoxib on 35 cases of knee osteoarthritis," Chinese Journal of Ethnomedicine and Ethnopharmacy, vol. 28, no. 10, pp. 106-109, 2019.

[40] L. Hong and D. Wu, "Observation of curative effect of Qufeng Jiangu recipe combined with glucosamine tablets on knee osteoarthritis," Zhejiang Journal of Integrated Traditional Chinese and Western Medicine, vol. 23, no. 3, pp. 207-208, 2013.

[41] J. Hu, L. Xue, D. Wang, and G. Li, "Clinical study on Bushen Zhuanggu decoction combined glucosamine hydrochloride for knee osteoarthritis," Shanghai Journal of Traditional Chinese Medicine, vol. 46, no. 6, pp. 55-57, 2012.

[42] L. Huang, X. Li, D. Chen, and Y. Liu, "Effect of tonifying kidney and TCM syndromes and celecoxib on curative effect and symptoms of knee osteoarthritis," Chinese Journal of Modern Drug Application, vol. 9, no. 11, pp. 182-183, 2015.

[43] Y. Jiang, "Clinical effect of Chinese herbs of strengthening kidney and activating blood on knee osteoarthritis," Chinese Journal of Traditional Medical Traumatology \& Orthopedics, vol. 17, no. 6, pp. 18-19, 2009.

[44] Y. Li, Z. Fan, and C. Lu, "Observation on the efficacy of Duhuozhitang combined with western medicine in treating knee osteoarthritis," Practical Clinical Journal of Integrated Traditional Chinese and Western Medicine, vol. 18, no. 4, pp. 146-148, 2018.

[45] H. Liu and W. Han, "Clinical observation on the treatment of knee osteoarthritis with Jingu Tongning Jiaonang combining glucosamine," Rheumatism and Arthritis, vol. 5, no. 5, pp. 21-23, 2016.

[46] W. Liu, "Clinical effect of Shujin decoction and Shirexibu combined with celecoxib on knee osteoarthritis and its effect on TNF- $\alpha$, VEGF and OPG," Journal of Sichuan of Traditional Chinese Medicine, vol. 35, no. 8, pp. 116-118, 2017.

[47] Y. Liu, "Clinical observation of Zhengqingfengtongning combined with glucose in the treatment of knee osteoarthritis," Hebei Medical Journal, vol. 33, no. 7, pp. 1082-1083, 2011.

[48] Y. Lu and J. Tan, "Lujiao Zhuanggu capsule combined with glucosamine hydrochloride tablets in treatment of early and mid-term stage osteoarthritis," Liaoning Journal of Traditional Chinese Medicine, vol. 43, no. 9, pp. 1903-1905, 2016.

[49] H. Luo and D. Hu, "Efficacy and safety of glucosamine hydrochloride combined with Sinomenine in the treatment of knee osteoarthritis," Chinese Traditional Patent Medicine, vol. 40, no. 3, pp. 708-710, 2019.

[50] Y. Ma, "Clinical observation of 32 cases on Duhuo Jisheng Tang in the treatment of knee osteoarthritis," Acta Chinese Medicine, vol. 33, no. 236, pp. 153-157, 2018.

[51] Y. Ma, "Therapeutic effect of Bushen Yiqi Huayu Jiedu decoction and glucosamine capsules on osteoarthritis," Journal of Mathematical Medicine, vol. 32, no. 5, pp. 732-733, 2019.

[52] Y. Ma, F. Xu, and H. Feng, "Clinical observation on 118 cases of knee osteoarthritis with nourishing liver and kidney and Huoxue Tongluo method," Clinical Journal of Traditional Chinese Medicine, vol. 21, no. 5, pp. 439-440, 2009.

[53] X. Mo, "Observation of curative effect of anti-bone hyperplasia capsule combined with celecoxib on knee osteoarthritis," Jiangxi Medical Journal, vol. 53, no. 12, pp. 1404-1406, 2018.

[54] J. Pan, W. Yang, J. Liu, H. Xie, K. Hong, and D. Guo, "Clinical efficacy and quality of life of knee osteoarthritis patients treated with Longbie capsule," Chinese Archives of Traditional Chinese Medicine, vol. 35, no. 3, pp. 558-561, 2017.

[55] Y. Qian, "Feasibility analysis of Yishen Quyu decoction for adjuvant treatment of knee osteoarthritis," Journal of Bethune Medical Science, vol. 17, no. 1, pp. 81-82, 2019.

[56] S. Ren, "Efficacy and prognosis of Duhuo Jisheng decoction for knee osteoarthritis," Journal of North Pharmacy, vol. 15, no. 11, pp. 111-112, 2018.

[57] X. Ren and D. Wang, "Observation on the effect of Yishen Quyu decoction on celecoxib in the treatment of acute knee osteoarthritis," Journal of Emergency in Traditional Chinese Medicine, vol. 25, no. 11, pp. 2191-2193, 2016.

[58] B. Rong and J. Li, "Clinical efficacy of Duhuo Jisheng Tang in treatment of patients with liver and kidney deficiency type knee osteoarthritis and changes in joint fluid metabolism," Chinese Journal of Experimental Traditional Medical Formulae, vol. 23, no. 23, pp. 159-165, 2017.

[59] Z. Shi, "Clinical observation of modified Duhuo Shengtang decoction combined with Western medicine in the treatment of liver and kidney deficiency knee osteoarthritis," Guangming Journal of Chinese Medicine, vol. 34, no. 12, pp. 1903-1904, 2019.

[60] S. Song and B. Li, "Observation of curative effect of Duhuoshengtang decoction combined with celecoxib on knee osteoarthritis," Practical Clinical Journal of Integrated Traditional Chinese and Western Medicine, vol. 17, no. 11, pp. 64$65,2017$.

[61] J. Sun, J. Sun, W. Wang, and Y. Cai, "Clinical effect observation of Qingbi decoction in treating Hanre Bizu type knee osteoarthritis," Clinical Journal of Traditional Chinese Medicine, vol. 30, no. 1, pp. 130-132, 2018.

[62] W. Tan, G. Li, and Q. Fu, "Clinical research of Astragalus Carapax Trionycis pill in the treatment of kidney deficiency type of knee osteoarthritis," China Medical Herald, vol. 11, no. 30, pp. 83-87, 2014.

[63] C. Tang and F. Li, "Clinical study on treatment of knee osteoarthritis with the method of Detoxificating and Incigorating the kidney," Chinese Archives of Traditional Chinese Medicine, vol. 28, no. 1, pp. 217-219, 2012.

[64] F. Wang, G. Wang, H. Zuo, T. Shen, X. Xu, and Y. Huang, "Preliminary reports of compound Rhizoma Corydalis Decumbentis combined with glucosamine hydrochloride in the treatment of prometaphase knee osteoarthritis," Chinese Journal of Modern Applied Pharmacy, vol. 30, no. 3, pp. 330332, 2013.

[65] X. Wang and W. Li, "Clinical observation on treatment of 45 cases of knee osteoarthritis with Taoren Jikang pill and celecoxib," Hunan Journal of Traditional Chinese Medicine, vol. 35, no. 2, pp. 63-65, 2019.

[66] X. Wen, M. Li, M. He, X. Cheng, J. Geng, and Y. Li, “Comparison of the curative effect between glucosamine hydrochloride and Angelicae Pubescentis and Loranthi decoction in the treatment of knee osteoarthritis," West China Medical Journal, vol. 31, no. 5, pp. 808-810, 2016.

[67] S. Wu, X. Hu, A. Chen, and C. Chen, "Clinical effect of Bushen Huoxue decoction combined with celecoxib on microinflammatory state and knee joint function in patients with knee osteoarthritis," Journal of Yunnan University of Traditional Chinese Medicine, vol. 41, no. 4, pp. 66-68, 2018.

[68] X. Wu, B. Liu, and Z. Fu, "Efficacy and safety evaluation of Sanqi Xuejie capsule combined with celecoxib in the treatment 
of knee osteoarthritis," Biped and Health, vol. 27, no. 18, pp. 151-152, 2018.

[69] Z. Wu, Y. Zhu, Y. Li, and F. Xu, "Comparative study on the curative effects and safety between ZHUANGGU TONGBI pill and glucosamine sulfate capsules in the treatment of knee osteoarthritis," The Journal of Traditional Chinese Orthopedics and Traumatology, vol. 24, no. 7, pp. 20-22, 2012.

[70] Q. Xia, P. Deng, and G. Lu, "Efficacy of glucosamine hydrochloride and Jinwu Gutong capsules in patients with knee osteoarthritis and its effect on inflammatory factor levels," AntiInfection Pharmacy, vol. 14, no. 9, pp. 1784-1786, 2017.

[71] J. Yang and C. Fan, "Effect of Jiawei Simiao powder on knee osteoarthritis and inflammatory factors," Global Traditional Chinese Medicine, vol. 9, no. 11, pp. 1360-1362, 2016.

[72] Z. Yang and Y. Liu, "Clinical efficacy of Duhuo Jisheng decoction combined with non-steroidal anti-inflammatory drugs in the treatment of knee osteoarthritis and its effect on articular cartilage," Journal of Liaoning University of Traditional Chinese Medicine, vol. 21, no. 11, pp. 218-221, 2019.

[73] S. Yao, Y. Zhang, and S. Liu, "Treatment of 41 cases of knee osteoarthritis with Duhuo Jisheng decoction and Western medicine," Shanxi Journal of Traditional Chinese Medicine, vol. 34, no. 11, pp. 1500-1501, 2013.

[74] C. Yi, Y. Zhou, W. Feng, and J. Li, "Clinical observation on Bushen Huoxue recipe combined with celecoxib in the treatment of knee osteoarthritis," Chinese Journal of Ethnomedicine and Ethnopharmacy, vol. 26, no. 2, pp. 121-122, 2016.

[75] M. Yi, "Clinical effect and possible mechanism of Qiangjinzhuangguyin recipe I combined with celecoxib in improving knee function in patients with knee osteoarthritis," Global Traditional Chinese Medicine, vol. 10, no. 8, pp. 977-980, 2017.

[76] J. Yu and H. Zhang, "Duhuo Jisheng Tang of knee osteoarthritis clinical observation," Chinese Journal of Experimental Traditional Medical Formulae, vol. 16, no. 7, pp. 215-217, 2010.

[77] J. Yuan, C. Luo, Y. Huang, and W. Liu, "Clinical study on oral application of self-made Bushen Huoxue Tang for treatment of early knee osteoarthritis," The Journal of Traditional Chinese Orthopedics and Traumatology, vol. 29, no. 8, pp. 26-32, 2017.

[78] C. Zhang and Z. Shi, "Efficacy of glucosamine hydrochloride combined with Jinwu Gutong capsule in the treatment of patients with knee osteoarthritis and its effect on serum inflammatory factors," Medical Journal of Chinese People's Health, vol. 28, no. 6, pp. 95-96, 2016.

[79] F. Zhang, "Analysis of the effect of Duhuozhitang on knee osteoarthritis," The Medical Forum, vol. 23, no. 25, pp. 36633664, 2019.

[80] J. Zhang, "Effects of total flavonoids of rhizome drynariae combined with glucosamine sulfate on symptoms and bone metabolism and inflammatory factors in patients with knee osteoarthritis," Chinese Journal of Osteoporosis, vol. 24, no. 7, pp. 884-887, 2018.

[81] L. Zhang, "Clinical study on Fugui Gutong capsules combined with celecoxib in treatment of senile knee osteoarthritis," Drugs \& Clinic, vol. 32, no. 9, pp. 1718-1721, 2017.

[82] C. Zheng, Y. Liu, and F. Zhang, "Clinical study of glucosamine sulfate combined with Zhengqingfengtongning in the treatment of knee osteoarthritis," Research of Integrated Traditional Chinese and Western Medicine, vol. 6, no. 3, pp. 123125, 2014.
[83] T. Zheng, Z. Xu, Z. Xu, and G. Zhang, "Clinical effect of Bushen Huoxue prescription on knee osteoarthritis," Chinese Archives of Traditional Chinese Medicine, vol. 37, no. 6, pp. 1506-1509, 2019.

[84] W. Zhong, H. Wu, Y. Li, and Z. Guo, "Observation on curative effect of Shentong Zhuyu decoction in the treatment of knee osteoarthritis with glucosamine hydrochloride," Asia-Pacific Traditional Medicine, vol. 13, no. 16, pp. 156-158, 2017.

[85] H. Zhou, "Clinical observation on the treatment of 43 cases of knee joint osteoarthritis with Bushen Qianjin Huoxue therapy," Guiding Journal of Traditional Chinese Medicine and Pharmacy, vol. 18, no. 2, pp. 112-113, 2012.

[86] F. Zhu, R. Zhou, Y. Shi, and Y. Tan, "Clinical study of Zhengqingfengtongning on knee osteoarthritis and its effect on cytokines," Contemporary Medicine, vol. 19, no. 12, pp. 1-3, 2013.

[87] S. Zhuo, "Clinical efficacy analysis and mechanism discussion of Rendong Bixie decoction on knee osteoarthritis," China Practical Medicine, vol. 14, no. 17, pp. 3-6, 2019.

[88] W. Zhang, S. Wang, R. Zhang et al., "Evidence of Chinese herbal medicine Duhuo Jisheng decoction for knee osteoarthritis: a systematic review of randomised clinical trials," BMJ Open, vol. 6, no. 1, 2016.

[89] C. V. Little and T. Parsons, "Herbal therapy for treating osteoarthritis," Cochrane Database of Systematic Reviews, no. 1, 2001.

[90] C. W. Cheng, T. X. Wu, H. C. Shang et al., "CONSORT extension for Chinese herbal medicine formulas 2017: recommendations, explanation, and elaboration (traditional Chinese version)," Annals of Internal Medicine, vol. 167, no. 2, pp. W7-W20, 2017.

[91] K. F. Schulz, D. G. Altman, and D. Moher, "CONSORT 2010 statement: updated guidelines for reporting parallel group randomized trials," Annals of Internal Medicine, vol. 152, no. 11, pp. 726-732, 2010.

[92] A. Flower, C. Witt, J. P. Liu, G. Ulrich-Merzenich, H. Yu, and G. Lewith, "Guidelines for randomised controlled trials investigating Chinese herbal medicine," Journal of Ethnopharmacology, vol. 140, no. 3, pp. 550-554, 2012.

[93] Z. X. Bian, H. Y. Tian, L. Gao et al., "Improving reporting of adverse events and adverse drug reactions following injections of Chinese materia medica," Journal of Evidence-Based Medicine, vol. 3, no. 1, pp. 5-10, 2010.

[94] J. Wang and X. Xiong, "Current situation and perspectives of clinical study in integrative medicine in China," EvidenceBased Complementary and Alternative Medicine, vol. 2012, Article ID 10.1155/2012/268542, 11 pages, 2012.

[95] A. Bensoussan, N. J. Talley, M. Hing, R. Menzies, A. Guo, and M. Ngu, "Treatment of irritable bowel syndrome with Chinese herbal Medicine," JAMA, vol. 280, no. 18, pp. 1585-1589, 1998. 